\title{
The High-Temperature Phase of Long-Range Spin Glasses
}

\author{
J. Fröhlich and B. Zegarlinski *
}

Theoretical Physics, ETH-Hönggerberg, CH-8093 Zürich, Switzerland

\begin{abstract}
We analyze the high-temperature phase of long-range Ising- and $N$ vector spin glasses with exchange couplings $\left\{J_{i j}\right\}, i, j \in Z^{d}$, which are independent random variables with $\bar{J}_{i j}=0$ and $\left|\bar{J}_{i j}^{p}\right| \leqq \gamma^{p} p !|i-j|^{-p \alpha d}, p=2,3, \ldots, \gamma$ is a finite constant and $\alpha>\frac{1}{2}$. We show that, for sufficiently high temperatures, the equilibrium state in the thermodynamic limit is (weakly) unique, and the quenched average of the square of connected correlations $\left\langle\sigma_{A} ; \sigma_{B}\right\rangle_{\beta}$ decays like $\operatorname{dist}(A, B)^{-2 \alpha d}$, despite of Griffiths singularities and the non-summable range of $J_{i j}\left(\right.$ for $\left.\frac{1}{2}<\alpha \leqq 1\right)$.
\end{abstract}

\section{Introduction: Problems, Notation, Main Results}

\subsection{Description of the Problems}

Real spin glasses are alloys of magnetic and non-magnetic, conducting materials, like iron and gold, manganese and copper. The magnetic atoms or ions (iron) are impurities in a non-magnetic material (gold). The magnetic properties of such a substance are approximately described by a classical spin system with long-range Ruderman-Kittel exchange interactions. We propose to analyze the behaviour of spin correlations in such systems at high temperatures.

Our methods are based on a sequence of high-temperature expansion steps followed by suitable upper bounds on the result of an individual expansion step. These upper bounds simplify the result of an expansion step and reduce the number of terms generated at the next expansion step. They are valid only on the real temperature axis, i.e. our methods only yield convergent bounds for sufficiently high, real temperatures, but divergent ones off the real axis. This feature permits us to avoid problems with Griffiths singularities which make full-fledged high-temperature expansions diverge for all or, at least, for very high temperatures, long before a transition temperature is approached.

* Permanent address: Institute of Theoretical Physics, University of Wrockaw, Wrocław, Poland 
As may be clear from these remarks, the analytical methods which we use to study the high-temperature behaviour of classical spin glasses are really quite robust. It is, for example, not particularly challenging to extend our methods to quantum mechanical spin glass models and to models with many-body interactions, but the notational complexity arising in such situations makes it appear unworthy to present details. A more interesting extension of our methods would be to study the regime where a large external magnetic field is applied to the system, but the temperature may be low. In this regime one also expects uniqueness of the equilibrium state and clustering of connected correlations. While we prove such results for systems in a magnetic field at high temperature, it is technically complicated to analyze what happens in a strong magnetic field at low temperatures, although there are no fundamental obstacles against doing just that.

The Hamiltonian of the spin glasses we wish to study in detail is thus given by

$$
H=H(\sigma)=-\sum_{i, j} J_{i j} \sigma_{i} \cdot \sigma_{j},
$$

where $\sigma_{i}$ is an Ising spin or a unit vector in $\mathbb{R}^{N}$ (an " $N$-vector"), with $N=2,3, \ldots$. In the Ruderman-Kittel ansatz [1], the positions of the magnetic atoms with spins $\sigma_{i}$ are the sites of some lattice, $\Gamma$, which we may choose for simplicity to be $Z^{d}$, but most sites remain empty (i.e. are occupied by a non-magnetic atom or ion). For $d=3$, the $R K K Y$ exchange couplings have the form

$$
J_{i j} \sim n_{i} n_{j} \cos \left(2 k_{F}|i-j|\right)|i-j|^{-3},
$$

where $\left\{n_{i}\right\}, i \in Z^{d}$, is a family of independent random variables with values 0 or 1 . (If $n_{i}=1$ site $i$ is occupied by a magnetic atom, if $n_{i}=0$ it is "empty.") The characteristic features of the system described by (1.1) and (1.2) are $\left.i \in Z^{d}\right)$;

(i) randomness (the dilution of magnetic atoms described by the variables $n_{i}$,

(ii) competition between ferromagnetic $\left(J_{i j}>0\right)$ and non-ferromagnetic $\left(J_{i j}<0\right)$ exchange couplings which produces frustration [2];

(iii) exchange couplings of very long, nonsummable range. (If $J_{i j}$ is chosen as in (1.2) then

$$
\overline{\sum_{j} J_{i j}}=\varrho^{2} \sum_{j} \cos \left(2 k_{F}|i-j|\right)|i-j|^{-3}<\infty, \quad \text { for } \quad k_{F} \neq 0,
$$

where $\varrho=\bar{n}_{i}$, but $\sum_{j}\left|J_{i j}\right|$ diverges almost surely.)

While we think that our methods may be applicable to spin glasses with $R K K Y$ exchange couplings we further simplify the problem, following Edwards and Anderson [3], by replacing the $R K K Y$ couplings by random exchange couplings $J_{i j}$ with the following properties:

(a) $\left\{J_{i j}\right\}$ is a family of independent random variables, with

(b) $\bar{J}_{i j}=0$, and

(c) $\left|\frac{j}{J_{i j}^{p}}\right| \leqq \gamma^{p} p !|i-j|^{-p \alpha d}, p=2,3, \ldots, 0<\gamma<\infty, \alpha>\frac{1}{2}$.

These properties retain the basic features (i)-(iii) described above, but simplify the probability theory. A typical example of a spin glass model of the kind we are 
able to analyze has exchange couplings, $J_{i j}$, given by

$$
J_{i j}=\mathscr{J}_{i j}|i-j|^{-\alpha d}, \quad \frac{1}{2}<\alpha \leqq 1,1
$$

where the variables $\mathscr{J}_{i j}$ are independent, identically distributed random variables with mean 0 and the property that

$$
\left|E \mathscr{J}_{i j}^{p}\right| \leqq \gamma^{p} p !, \quad p=2,3, \ldots,
$$

and we now denote expectations with respect to $\left\{\mathscr{J}_{i j}\right\}$ by the symbol $E$. All our results hold for the general class of exchange couplings, $J_{i j}$, described in (a)-(c), above, but we find it convenient to present our analysis for the examples specified in (1.3), (1.4).

The study of spin glasses, especially long-range spin glasses, is an important task, not only in order to understand the magnetic alloys they were invented for, but also because of applications to models of neural networks and associative memory [4] or to problems in stochastic optimization [5]. Unfortunately, there are only few rigorous results on spin glasses. They are among the most subtle systems encountered in equilibrium statistical mechanics. There are no rigorous results about the low-temperature behaviour of spin glasses, although a good theory for the low-temperature behaviour of the Sherrington-Kirkpatrick model [6] is available which may be exact but is certainly non-rigorous [7], and a decent heuristic picture of the low-temperature behaviour of the nearest-neighbor Edwards-Anderson Ising spin glasses has been developed $[8,9]$ which is partly based on numerical experiments, partly on heuristic, analytical arguments.

Surprisingly, not even the high-temperature disordered or large-magnetic field phase of spin glasses has been studied mathematically, until recently. In [10] this problem was tackled for short-range spin glasses, while in [11] we have started to investigate long-range spin glasses. Results on the absence of transitions and/or ordering may be found in [12] for one-dimensional, long-range Ising spin glasses and in [13] for two-dimensional long-range spin glasses with continuous internal symmetry groups. In this paper we elaborate on the methods and results in [11] and extend them to a wider range of problems. The difficulties which we encounter in our analysis are connected with the following properties of spin glasses.

(A) Griffiths Singularities [14]. There exist, with probability one, arbitrarily large, connected regions, $\Lambda$, in the lattice with the property that $J_{i j}$ is anomalously large and positive, for $i$ and $j$ in $\Lambda$, but $\left|J_{i j}\right|$ is small for $i \in \Lambda, j \notin \Lambda$. The contributions of the spins in $\Lambda$ to the free energy of the system are believed to yield singularities of the free energy in the complex $\beta$-plane off the real axis, but arbitrarily close to the real axis. (Here $\beta$ denotes the inverse temperature.) If the range of values of $J_{i j}$ is unbounded such singularities may be arbitrarily close to the origin.

(B) Long-Range Interactions. There exist, with probability one, arbitrarily far distant, arbitrarily large, finite connected sets, $\Lambda_{1}$ and $\Lambda_{2}$, in the lattice with the property that $J_{i j}>$ const $|i-j|^{-\alpha d}$, for $i \in \Lambda_{1}$ and $j \in \Lambda_{2}$, but $\left|J_{i j}\right|$ is small if $i \in \Lambda_{1} \cup \Lambda_{2}$,

${ }^{1}$ If $\alpha>1$ and $\left|\mathscr{F}_{i j}\right|$ is bounded then standard high-temperature expansions converge, for $|T|$ large enough. See also [10] 
and $j \notin \Lambda_{1} \cup \Lambda_{2}$. The contributions of spins in $\Lambda_{1} \cup \Lambda_{2}$ to the free energy are believed to have singularities in $\beta$ which, though off the real axis, may be arbitrarily close to $\beta=0$.

Griffiths' original result [14] does not quite prove the correctness of (A) and (B) but strongly suggests that these statements are indeed valid. As a consequence we strongly expect that full-fledged high-temperature expansions diverge for arbitrary $|\beta|$, or at least long before the transition point is reached. The purpose of this article is to describe methods which overcome this difficulty and permit us to analyze the properties of long-range spin glasses at high temperatures in detail. Our methods are based on an iteration of high-temperature expansion steps followed by upper bounds, only valid on the real $\beta$-axis, which reduce the number of terms generated by the expansion step.

Next, we introduce our notation and then summarize the main results of this paper.

\subsection{A List of Notations}

1) $\Gamma$ denotes a periodic $d$-dimensional lattice, typically $Z^{d}$; $d$ arbitrary. The symbol $\Lambda \nearrow \Gamma$ indicates that $\Lambda$ increases to the entire lattice $\Gamma$ through a family, $\left\{\Lambda_{n}\right\}_{n=0}^{\infty}$, of subsets with the following properties: Every $\Lambda_{n}$ is bounded; $\Lambda_{n} \subset \Lambda_{n+1}$, for all $n=0,1,2, \ldots$; every bounded subset $\Lambda \subset \Gamma$ is contained in a set $\Lambda_{n}$, for some finite $n$.

2) A configuration of spins on $\Gamma$ is given by a family $\left\{\sigma_{i}\right\}_{i \in \Gamma}$ of spins, $\sigma_{i}$, belonging to

$$
S_{N}=\left\{\sigma \equiv\left(\sigma^{1}, \ldots, \sigma^{N}\right): \sum_{m=1}^{N}\left(\sigma^{m}\right)^{2}=1\right\},
$$

with $S_{1} \equiv\{1,-1\}$ for Ising spins. It is natural to also include the point $\sigma=0$, defining $S_{N} \equiv S_{N} \cup\{0\}$. This will permit us to impose 0 -boundary conditions (b.c.) on subsets of $\Gamma$. The set of all spin configurations $\left(S_{N}^{\circ}\right)^{\times \Gamma}$, is denoted by $\Omega$. For $\sigma \in \Omega$ and $\Lambda \in \Gamma, \sigma_{\Lambda}$ denotes the restriction of $\sigma$ to $\Lambda$, i.e.

$$
\sigma_{\Lambda}=\left\{\sigma_{i}: i \in \Lambda, \sigma \in \Omega\right\}, \text { and }\left(\sigma_{\Lambda_{1}}, \sigma_{\Lambda_{2}}\right)=\left\{\sigma_{i}: i \in \Lambda_{1} \cup \Lambda_{2}, \sigma \in \Omega\right\}
$$

Let $A$ denote the graph of a function from $\Gamma \times\{1, \ldots, N\}$ to $\{0,1,2, \ldots\}$, and let $\bar{A}$ be the projection of $A$ onto $\Gamma$. We define

$$
\sigma_{A} \equiv \prod_{i \in \bar{A}} \prod_{m=1}^{N}\left(\sigma_{i}^{m}\right)^{n(m, i)},
$$

with $\{(i, m, n(m, n)): i \in \bar{A}, m=1, \ldots, N\}=A$. For Ising spins, $N=1$ and $\left(\sigma_{i}\right)^{2}=1$, so we may identify $A$ with $\bar{A}$. In this case, $\Omega$ is a group, with $\sigma \cdot \tilde{\sigma}$ defined by $(\sigma \tilde{\sigma})_{i}=\sigma_{i} \tilde{\sigma}_{i}$, for $\sigma$ and $\tilde{\sigma}$ in $\Omega$.

We let $\Sigma_{\Lambda}$ denote the $\sigma$-algebra generated by $\left\{\sigma_{i}^{m}: m=1, \ldots, N, i \in \Lambda\right\}$, for any subset $\Lambda \subset \Gamma$, and $\Sigma=\vee_{\Lambda}{ }_{\Gamma} \Sigma_{\Lambda}$. The "local observables" of the system are the $\Sigma$ measurable functions on $\Omega$.

3) States of the system are probability measures, $d \mu$, on the measure space $(\Omega, \Sigma)$. States of finite subsystems are probability measures on $\left(\Omega, \Sigma_{\Lambda}\right)$, where $\Lambda$ is a finite subset of $\Gamma$. Let $d \varrho$ be a probability measure on $S_{N}$, and let $d \varrho\left(\sigma_{\Lambda}\right)$ be some probability measure on $\left(S_{N}^{\circ}\right)^{\times|\Lambda|}$. An example of a state for a finite subsystem 
in $\Lambda^{\prime}$ is given by

$$
d \mu_{0 \varrho}\left(\sigma_{\Lambda^{\prime}}\right)=d \varrho\left(\sigma_{\Lambda^{\prime} \backslash \Lambda}\right) \prod_{i \in \Lambda} d \varrho\left(\sigma_{i}\right) .
$$

Let $H$ be given by (1.1). We set

$$
H\left(\sigma_{\Lambda}\right) \equiv H^{\mathscr{g}}\left(\sigma_{\Lambda}\right):=-\sum_{i, j \in \Lambda} J_{i j} \sigma_{i} \sigma_{j},
$$

with $J_{i j}=\mathscr{J}_{i j}|i-j|^{-\alpha d}, \alpha>\frac{1}{2}$, where the couplings $\mathscr{J}_{i j}$ are random variables as in (1.3), (1.4). An equilibrium state for a spin glass at inverse temperature $\beta$ in a finite region $\Lambda \subset \Gamma$ with b.c. given by $d \varrho\left(\sigma_{\Lambda^{\prime} \backslash \Lambda}\right)$ is given by

$$
d \mu_{\Lambda \underline{\varrho}}(\cdot) \equiv d \mu_{\Lambda \underline{\underline{\varrho}}}^{\beta \mathscr{q}}(\cdot):=\frac{\mu_{0 \varrho}\left(e^{-\beta H^{\mathscr{g}}\left(\sigma_{\Lambda^{\prime}}\right)} \cdot\right)}{\mu_{0 \underline{\varrho}}\left(e^{-\beta H^{\mathscr{F}}\left(\sigma_{\Lambda^{\prime}}\right)}\right)} .
$$

Here $\mu(F) \equiv \mu F=\int_{\Omega} F(\sigma) d \mu(\sigma)$. Connected expectations are defined by

$$
\mu(F ; G) \equiv \mu(F G)-\mu(F) \mu(G) .
$$

If $d \varrho\left(\sigma_{\Lambda^{\prime} \backslash \Lambda}\right) \equiv \prod_{i \in \Lambda^{\prime} \backslash \Lambda} \delta_{0}\left(\sigma_{i}\right) d^{N} \sigma_{i}$, we use the shorthand notation

$$
d \mu_{\Lambda \varrho}(\cdot) \equiv d \mu_{\Lambda}(\cdot)
$$

Let $C$ denote some condition imposed on a subset of the couplings $\left\{J_{i j}\right\}_{i, j \in \Gamma}$. By

$$
\left.\mu_{\Lambda \underline{\underline{\varrho}}}(\cdot)\right|_{C} \equiv\left[\mu_{\Lambda \underline{\underline{\varrho}}}(\cdot)\right]_{C}
$$

we mean the state defined in (1.10), but with the couplings $\left\{J_{i j}\right\}$ satisfying condition $C$. The operation $\left.(\cdot)\right|_{C} \equiv[(\cdot)]_{C}$ is extended to sums and products of expectations in the equilibrium states $\mu_{\Lambda \varrho}(\cdot)$ by applying them to each factor in each summand. For example

$$
\left.\left.\mu(F ; G)\right|_{C} \equiv \mu(F G)\right|_{C}-\left.\left.\mu(F)\right|_{C} \mu(G)\right|_{C}
$$

Let

$$
K_{i j} \equiv \beta J_{i j}, \quad S_{i j} \equiv s_{i j} \beta J_{i j}, \quad s_{i j} \in[0,1] .
$$

If the condition $C$ corresponds to

$$
\left\{K_{i j}=0, i \in A, j \in B, \beta J_{i j} \rightarrow S_{i j}, i \in D, j \in E\right\},
$$

we also use the notation

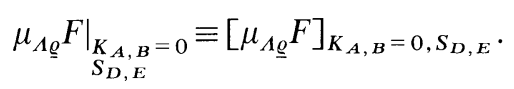

Here $A, B, D$, and $E$ are subsets of $\Gamma$.

A derivative with respect to the variable $S_{i j}$ or $S_{i j}^{\prime}\left(\equiv s_{i j}^{\prime} \beta J_{i j}\right)$ is denoted by $\partial_{i j}$.

4) The joint expectation with respect to the random couplings $\left\{\mathscr{J}_{i j}\right\}_{i, j \in \Gamma}$ is denoted by $E$. As in (1.3), (1.4) we assume $E$ to be given by a product measure, with $E\left(\mathscr{J}_{i j}\right)=0$, for all $i$ and $j$, and

$$
\left|E\left(\mathscr{J}_{i j}^{p}\right)\right| \leqq \gamma^{p} p !, \quad p=2,3, \ldots,
$$


for some finite constant $\gamma$. It will always be assumed that b.c. $\underline{\varrho}$ are independent of the random couplings. This assumption as well as our assumptions on the nature of the couplings $J_{i j}$ could be weakened somewhat, but that would complicate our notations and expansion steps in a rather awkward manner.

Constants in inequalities are denoted by $K, K^{\prime}, D_{\beta}, \bar{D}_{\beta}, \ldots$. Their values may change from one estimate to another one. Restrictions on $\beta$ are indicated by $0 \leqq \beta<\beta_{0}$, where $\beta_{0}$ is a positive constant whose value may vary from one estimate to another one.

5) Prominent special quantities are:

- the Edwards-Anderson order parameter

$$
q_{E A}(\beta)=\sup _{\underline{\varrho}} \varlimsup_{\Lambda \nearrow \Gamma} E\left(\mu_{\Lambda \varrho} \sigma_{0}\right)^{2}
$$

- the spin-spin correlation $\mu_{\Lambda \varrho}\left(\sigma_{0} ; \sigma_{j}\right)$, the quenched correlations

$$
E \mu\left(\sigma_{0} ; \sigma_{j}\right)^{2}=\lim _{\Lambda \nearrow \Gamma} E \mu_{\Lambda \underline{\varrho}}\left(\sigma_{0} ; \sigma_{j}\right)^{2}
$$

and the "susceptibility"

$$
\chi^{(2)}(\beta)=\sum_{j \in \Gamma} E \mu\left(\sigma_{0} ; \sigma_{j}\right)^{2}
$$

It will be shown that, in the limit $\Lambda \nearrow \Gamma$, these quantities are independent of the b.c. $\varrho$, for $\beta \geqq 0$ small enough.

In [11] we have shown that, for Ising spin glasses at sufficiently high temperatures, with $\alpha>\frac{1}{2}$ and $d \varrho(\sigma)$ symmetric in $\sigma$,

(1) $q_{E A}(\beta)=0$,

(2) $\chi^{(2)}(\beta)<\infty$, and

(3) $E \mu\left(\sigma_{0} ; \sigma_{j}\right)^{2} \sim|j|^{-2 \alpha d}$, as $|j| \rightarrow \infty$.

The purpose of this paper is to analyze the existence and uniqueness of the thermodynamic limit of the states $\mu_{\Lambda \varrho}$ and their cluster properties for general Isingand $N$-vector spin glasses at high temperatures and in an arbitrary magnetic field.

\subsection{Main Results and Contents of Further Sections}

In Sect. 2, we analyze Ising spin glasses, as these models are somewhat simpler than general $N$-vector spin glasses and yet illustrate all basic features of our method. We always assume that the exchange couplings $\left\{\mathscr{J}_{i j}\right\}$ are as in (1.3), (1.4), with $\alpha>\frac{1}{2}$. The single spin distribution $d \varrho(\sigma)$ is arbitrary, but fixed, and the boundary conditions, $d \underline{\varrho}$, are independent of $\left\{\mathscr{J}_{i j}\right\}$.

In Sect. 2.1 we prove

Theorem 1 (Existence and Uniqueness). If $0 \leqq \beta<\beta_{0}$, for some sufficiently small $\beta_{0}>0$, then

$$
\lim _{\Lambda \nearrow \Gamma} E\left(\mu_{\Lambda \varrho} \sigma_{A}-\mu_{\Lambda \varrho^{\prime}} \sigma_{A}\right)^{2}=0,
$$

for any bounded $A \subset \Gamma$. For fixed $A$, the convergence is uniform in the choice of

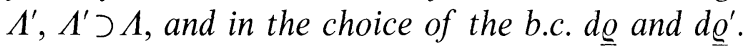


For a suitably chosen sequence of finite regions $\left\{\Lambda_{n}\right\}_{n=0}^{\infty}$ increasing to $\Gamma$,

$$
\lim _{n \rightarrow \infty} \mu_{\Lambda_{n} \underline{\underline{Q}}}=: \mu
$$

exists and is independent of the b.c. d@, E-almost surely.

In Sect. 2.2, we establish cluster properties. We prove

$$
E\left(\mu_{\Lambda}\left(\sigma_{A} ; \sigma_{B}\right)^{2}\right) \leqq \beta^{2} \bar{C}_{\beta} d(A, B)^{-2 \alpha d},
$$

where $\bar{C}_{\beta}$ is a finite constant independent of $\Lambda$ (but depending on $|A|$ and $|B|$, with $|A|=$ cardinality of $A)$, and $d(A, B)$ is the Euclidean distance between $A$ and $B$.

For $A=\{i\}, B=\{j\}$, we can also prove that

$$
E\left(\mu_{\Lambda}\left(\sigma_{i} ; \sigma_{j}\right)^{2}\right) \geqq \beta^{2} \underline{C}_{\beta} d(i, j)^{-2 \alpha d},
$$

where $\underline{C}_{\beta}$ is a finite constant independent of $\Lambda$, provided $\beta$ is small enough. Hence

$$
E\left(\mu\left(\sigma_{i} ; \sigma_{j}\right)^{2}\right) \sim \beta^{2} d(i, j)^{-2 \alpha d},
$$

as $d(i, j) \rightarrow \infty$. Lower bounds of the form (1.19) are expected to hold true for general, bounded sets $A$ and $B$, but the complexity of the expansions necessary to establish them makes it a rather unappetizing task to provide details.

In Sect. 3, we extend our results for Ising spin glasses to $N$-vector spin glasses. To accomplish that purpose we develop a more general, abstract inductive construction, organizing terms in trees. In certain respects our generalized (inductive) tree expansion is simpler to grasp than the more explicit constructions used in the Ising model. It is, however, less explicit and makes higher demands on abstract reasoning. This is why we felt it worthwhile to first treat the Ising spin glass in detail.

In Sect. 4, we sketch some extensions of our methods, e.g. to proving asymptoticity of the high-temperature expansion, propose some open problems and draw our conclusions. It may be appropriate to emphasize that our expansions incorporate some general principles which are applicable in a much wider context than the one considered in this paper. We feel that they can be used quite generally to convert asymptotic, but possibly divergent expansions in statistical mechanics and quantum field theory into convergent estimates. It might be interesting to try this out on perturbation theory for the vertex functions of lattice $\lambda \varphi_{d}^{4}$ theory in $d>4$ dimensions.

Some important technical estimates are collected in two appendices.

\section{The High-Temperature Phase of Ising Spin Glasses}

In this section we present a detailed analysis of the high-temperature phase of longrange Ising spin glasses. We prove existence and uniqueness of the thermodynamic limit and cluster properties of the equilibrium state at high temperatures. Our results can be used to show that the standard high-temperature expansion for the free energy or for correlations is asymptotic. 
2.1. Existence and Uniqueness of the Thermodynamic Limit

Let $\Lambda, \Lambda^{\prime}$ be finite subsets $\Gamma$, with $\Lambda^{\prime} \supset \Lambda$. We define a state

$$
d \mu_{0 \varrho}\left(\sigma_{\Lambda^{\prime}}\right)=d \underline{\varrho}\left(\sigma_{\Lambda^{\prime} \backslash \Lambda}\right) \prod_{j \in \Lambda} d \varrho\left(\sigma_{j}\right)
$$

where $d \varrho$ is an arbitrary probability measure on $\{-1,1\}$ which is kept fixed throughout Sect. 2, and $d \underline{\underline{ }}\left(\sigma_{\Lambda^{\prime} \backslash \Lambda}\right)$ is an arbitrary probability measure on $\{-1,0,1\}^{\times\left|\Lambda^{\prime} \backslash \Lambda\right|}$ whose role will be, for example, to specify boundary conditions in $\Lambda^{\prime} \backslash \Lambda$. We shall always assume that the measure $d \varrho$ is independent of $\left\{\mathscr{J}_{i j}\right\}_{i \in \Lambda, j \in \Lambda^{\prime}}$. The point of adding 0 to the state space of each $\sigma_{i}, \dot{i} \in \Lambda^{\prime} \backslash \Lambda$, is that this permits us to impose 0 b.c. in $\Lambda^{\prime} \backslash \Lambda$.

We define finite-volume equilibrium states $\mu_{\Lambda \varrho}$ with b.c. in $\Lambda^{\prime} \backslash \Lambda$ specified by $d \varrho\left(\sigma_{\Lambda^{\prime} \backslash \Lambda}\right)$ by

where

$$
\mu_{\Lambda \underline{\varrho}}(\cdot) \equiv \mu_{\Lambda \underline{\rho}}^{\beta \mathscr{g}}(\cdot):=\frac{\mu_{0 \underline{\varrho}}\left(e^{-\beta H\left(\sigma_{\Lambda^{\prime}}\right)} \cdot\right)}{\mu_{0 \underline{\underline{\varrho}}}\left(e^{-\beta H\left(\sigma_{\Lambda^{\prime}}\right)}\right)},
$$

$$
H\left(\sigma_{\Lambda^{\prime}}\right) \equiv H^{\mathscr{I}}\left(\sigma_{\Lambda^{\prime}}\right):=-\sum_{i, j \in \Lambda^{\prime}} \frac{\mathscr{J}_{i j}}{|i-j|^{\alpha d}} \sigma_{i} \sigma_{j},
$$

for some $\alpha>\frac{1}{2}$. By choosing

$$
d \underline{\varrho}\left(\sigma_{\Lambda^{\prime} \backslash \Lambda}\right)=\prod_{j \in \Lambda^{\prime} \backslash \Lambda} \delta_{0}\left(\sigma_{j}\right) d \sigma_{j},
$$

we can impose 0 - b.c. on $\Lambda^{c}$.

We set

$$
\sigma_{A}:=\prod_{i \in A} \sigma_{i}
$$

Our first result is the following

Proposition 2.1. If $0 \leqq \beta<\beta_{0}$, for some sufficiently small $\beta_{0}$, then

$$
\lim _{\substack{\Lambda \rtimes \Gamma \\ \Lambda^{\prime} \supset \Lambda}} E\left(\mu_{\Lambda \varrho \underline{\varrho}} \sigma_{A}-\mu_{\Lambda \underline{\varrho}^{\prime}} \sigma_{A}\right)^{2}=0,
$$

for any bounded $A \subset \Gamma$. For fixed $A$ and fixed $d \varrho\left(\sigma_{i}\right), i \in \Lambda$, the convergence is uniform in the choice of $\Lambda^{\prime}, \Lambda^{\prime} \supset \Lambda$, and in the choice of the probability measures $d \varrho\left(\sigma_{\Lambda^{\prime} \backslash \Lambda}\right)$, $d \varrho^{\prime}\left(\sigma_{\Lambda^{\prime} \backslash \Lambda}\right)$.

From this proposition we get

Corollary 2.2. Under the hypotheses of Proposition 2.1,

$$
\lim _{\Lambda \nearrow \Gamma} \mu_{\Lambda \underline{\underline{Q}}}=: \mu
$$

exists and is independent of the b.c. imposed by $d \underline{\varrho}\left(\sigma_{\Lambda \wedge \Lambda}\right)$, E-almost surely.

Proof of Corollary 2.2. We choose sets $\Lambda$ and $\Lambda^{\prime} \supset \Lambda$ and set

and

$$
\left.\begin{array}{l}
d \varrho\left(\sigma_{\Lambda^{\prime} \backslash \Lambda}\right)=\prod_{i \in \Lambda^{\prime} \backslash \Lambda} d \varrho\left(\sigma_{i}\right) \\
d \varrho^{\prime}\left(\sigma_{\Lambda^{\prime} \backslash \Lambda}\right)=\prod_{i \in \Lambda^{\prime} \backslash \Lambda} \delta_{0}\left(\sigma_{i}\right) d \sigma_{i} .
\end{array}\right\}
$$


By Proposition 2.1, for any bounded $A$ and arbitrary $\varepsilon>0$,

$$
E\left(\mu_{\Lambda \underline{\underline{\underline{e}}}} \sigma_{A}-\mu_{\Lambda \underline{\underline{Q}}} \sigma_{A}\right)^{2}<\varepsilon
$$

if $\Lambda$ is sufficiently large, uniformly in $\Lambda^{\prime} \supset \Lambda$. Since, by (2.2) and (2.5), $\mu_{\Lambda \underline{\underline{Q}}}=\mu_{\Lambda^{\prime}}$, and $\mu_{\Lambda \underline{Q}^{\prime}}=\mu_{A}$, see (1.2), we conclude from (2.6) that

$$
\lim _{\Lambda>\Gamma} \mu_{A \underline{Q}^{\prime}} \sigma_{A} \equiv \lim _{A>\Gamma} \mu_{A} \sigma_{A}=: \mu \sigma_{A}
$$

exists $E$-almost surely, for a suitably chosen sequence of sets $\Lambda$ increasing to $\Gamma$. Using Proposition 2.1 again, we see that, for any $\varepsilon>0$,

$$
E\left(\mu_{A} \sigma_{A}-\mu_{\Lambda \varrho} \sigma_{A}\right)^{2}<\varepsilon
$$

for an arbitrary choice of $d \varrho\left(\sigma_{\Lambda^{\prime} \backslash \Lambda}\right)$, uniformly in $\Lambda^{\prime}$, provided $\Lambda$ is large enough. This proves uniqueness and independence of b.c. of the limiting state $\mu, E$-almost surely. We remark that convergence holds, a priori, in $L^{2}\left(\left\{\mathscr{J}_{i j}\right\}, d E\right)$, but by passing to diagonal subsequences of sets $A$ increasing to $\Gamma$ we obtain $E$-almost-sure convergence, for arbitrary bounded subsets $A \subset \Gamma$.

We now turn to the proof of Proposition 2.1.

We choose a bounded subset $A$ of $\Gamma$. Given $A$, we introduce some ordering, $\prec$, of the sites in $\Gamma$ and in $\Gamma^{c}$ such that the sites in $A$ are smaller (with respect to $\prec$ ) than the sites in $\Lambda \backslash A$. Further properties of $\prec$ will be specified where needed. Let $k_{0}$ be the smallest site in $A$. We now perform a sequence of expansion steps in the couplings $J_{k_{0} j}, j \in \Lambda^{c}$.

$$
\begin{aligned}
& E\left(\mu_{\Lambda \underline{\varrho}} \sigma_{A}-\mu_{\Lambda \underline{\varrho}^{\prime}} \sigma_{A}\right)^{2} \\
& =\left.E\left(\mu_{\Lambda \varrho} \sigma_{A}-\mu_{\Lambda \underline{Q}^{\prime}} \sigma_{A}\right)^{2}\right|_{K_{k_{0} \Lambda^{c}}=0}+\sum_{j \in \Lambda^{\prime} \backslash \Lambda} \beta^{2}\left|k_{0}-j\right|^{-2 \alpha d} \int_{0}^{1} d s_{k_{0} j} \int_{0}^{s_{k o j}} d s_{k_{0} j}^{\prime} \\
& \times E\left(\mathscr{J}_{k_{0 j} j}^{2}\left[\partial_{k_{0} j}^{2}\left(\mu_{\Lambda \underline{\underline{Q}}} \sigma_{A}-\mu_{\Lambda \underline{\underline{Q}}{ }^{\prime}} \sigma_{A}\right)^{2}\right]_{K_{K_{k_{0} j}}=0, i \prec j}\right)^{2},
\end{aligned}
$$

where $i \in \Lambda^{\prime} \backslash \Lambda, i<j$, in the last factor on the r.h.s. of (2.8). In the derivation of (2.8) we have used the symmetry of $E$ which guarantees that

$$
\beta\left|k_{0}-j\right|^{-\alpha d} E\left(\mathscr{J}_{k_{0} j}\left[\partial_{k_{0} j}\left(\mu_{\Lambda \underline{\underline{\varrho}}} \sigma_{A}-\mu_{\Lambda \underline{\underline{Q}}} \sigma_{A}\right)^{2}\right]_{\underline{k_{k_{0}}}}=0\right)=0 .
$$

(We also recall that $\partial_{i j} \equiv \frac{\partial}{\partial S_{i j}}$.) Since the factor

$$
\left[\partial_{k_{0} j}^{2}\left(\mu_{\Lambda \underline{\underline{\rho}}} \sigma_{A}-\mu_{\Lambda \underline{\underline{\rho}}^{\prime}} \sigma_{A}\right)^{2}\right] \ldots
$$

is bounded uniformly in $\Lambda, \Lambda^{\prime}, A, \varrho, \varrho^{\prime}$ and in the conditions, $[(\cdot)] \ldots$, imposed on coupling constants, we obtain from (2.8)

$$
\begin{aligned}
I_{A} & \equiv E\left(\mu_{\Lambda \underline{\underline{Q}}} \sigma_{A}-\mu_{\Lambda \underline{\underline{Q}}^{\prime}} \sigma_{A}\right)^{2} \\
& \leqq\left. E\left(\mu_{\Lambda \underline{\underline{\varrho}}} \sigma_{A}-\mu_{\Lambda \underline{\underline{Q}}^{\prime}} \sigma_{A}\right)^{2}\right|_{K_{k_{0} \Lambda^{c}}=0}+a \beta^{2} \sum_{j \in \Lambda^{\prime} \backslash \Lambda}\left|k_{0}-j\right|^{-2 \alpha d},
\end{aligned}
$$

for some finite constant $a>0$, independent of $\beta$ and "everything else."

Next, we note that $(2.8)-(2.10)$ do not depend on special choices of the couplings, $J_{i j}$, i.e. we can apply expansion steps, like (2.8)-(2.10), to the first term on 
the r.h.s. of (2.10), i.e. to

$$
\left.I_{A}\left(K_{k_{0} \Lambda^{c}}=0\right) \equiv E\left(\mu_{\Lambda \varrho} \sigma_{A}-\mu_{\Lambda \underline{\varrho}^{\prime}} \sigma_{A}\right)^{2}\right|_{K_{k_{0} \Lambda^{c}}=0} .
$$

Hence

$$
\begin{aligned}
I_{A}\left(K_{k_{0} \Lambda^{c}}=0\right)= & I_{A}\left(K_{k_{0} \Gamma}=0\right)+\sum_{k_{1} \in \Lambda \backslash k_{0}} \beta^{2}\left|k_{0}-k_{1}\right|^{-2 \alpha d} \\
& \times \int_{0}^{1} d s_{k_{0} k_{1}} \int_{0}^{s_{k_{0} k_{1}}} d s_{k_{0} k_{1}}^{\prime} E\left(\mathscr{J}_{k_{0} k_{1}}^{2}\left[\partial_{k_{0} k_{1}}^{2}\left(\mu_{\Lambda \underline{\varrho}} \sigma_{A}-\mu_{\Lambda \underline{\varrho}^{\prime}} \sigma_{A}\right)^{2}\right]_{C_{k_{0^{\prime}} k_{1}}}\right),
\end{aligned}
$$

where $C_{k_{0} k_{1}}$ is the condition that $K_{k_{0} \Lambda^{c}}=0, K_{k_{0} i}=0$, for all $i \prec k_{1}$, and $\beta J_{k_{0} k_{1}}$ is replaced by $S_{k_{0} k_{1}}^{\prime}=s_{k_{0} k_{1}}^{\prime} \beta J_{k_{0} k_{1}}$.

If $d \varrho(\sigma)$ is symmetric then

$$
\left.\mu_{\Lambda \varrho} \sigma_{A}\right|_{K_{k_{0}} \Gamma}=\left.\mu_{\Lambda \underline{\varrho}^{\prime}} \sigma_{A}\right|_{K_{k_{0} \Gamma}}=0
$$

i.e. $I_{A}\left(K_{k_{0} \Gamma}=0\right)$ vanishes. This would somewhat simplify our analysis. Otherwise, $I_{A}\left(K_{k_{0} \Gamma}=0\right)$ needs to be reexpanded by repeating the same steps as above, for all sites $k \in A$. Then we may use that

$$
\left(\mu_{\Lambda \underline{\underline{\varrho}}} \sigma_{A}-\mu_{\Lambda \underline{\underline{\underline{\varrho}}}} \sigma_{A}\right)_{K_{A \Gamma}}=0
$$

and the expansion will terminate. Hence we shall apply an identity analogous to (2.11) to all terms $I_{A}\left(K_{A_{n} \Gamma}=0\right)$, for a sequence $\left\{A_{n}\right\}$ of subsets of $A$ increasing, with respect to the order $\prec$, to $A$. The index $n$ ranges from 1 to $|A|$, with $A_{1}=k_{0}$, $A_{2}=\left\{k_{0}, k_{1}^{\prime}\right\}$, where $k_{1}^{\prime}$ is the least site $\succ k_{0}$, etc. All these terms will generate terms like the second term on the r.h.s. of (2.11), but with slightly different conditions, $C_{A_{n} k_{1}}$, replacing $C_{k_{0} k_{1}}$, imposed on the exchange couplings.

In order to make progress, we must find an appropriate upper bound on

$$
\left|\left[\partial_{i j}^{2}\left(\mu_{\Lambda \varrho} \sigma_{B}-\mu_{\Lambda \varrho^{\prime}} \sigma_{B}\right)^{2}\right]_{C}\right|,
$$

for arbitrary finite subsets $B$ of $\Gamma$, arbitrary $\Lambda, \varrho, \varrho^{\prime}$, and $C$. In Appendix A.1 we prove

$$
\begin{aligned}
& \left|\left[\partial_{i j}^{2}\left(\mu_{\Lambda \varrho} \sigma_{B}-\mu_{\Lambda \varrho^{\prime}} \sigma_{B}\right)^{2}\right]_{C}\right| \\
& \leqq K\left[\left(\mu_{\Lambda \varrho} \sigma_{B}-\mu_{\Lambda \underline{\underline{\varrho}}} \sigma_{B}\right)^{2}+\left(\mu_{\Lambda \varrho \underline{\varrho}} \sigma_{B} \sigma_{i} \sigma_{j}-\mu_{\Lambda \varrho^{\prime}} \sigma_{B} \sigma_{i} \sigma_{j}\right)^{2}+\left(\mu_{\Lambda \varrho} \sigma_{i} \sigma_{j}-\mu_{\Lambda \varrho^{\prime}} \sigma_{i} \sigma_{j}\right)^{2}\right]_{C},
\end{aligned}
$$

for some finite constant $K>0$.

Remark. If $|A|$ is odd and $d \varrho$ is symmetric in $\sigma$, the last term on the r.h.s. of $(2.13)$ can be omitted, and there will occur further simplifications.

We now insert (2.13) on the r.h.s. of (2.11) and then perform a Taylor expansion in $S_{k_{0} k_{1}}^{\prime}$ to second order, apply (2.13) again to the resulting terms, and so on. This yields the following upper bound:

$$
\begin{aligned}
& \sum_{k_{1} \in \Lambda \backslash k_{0}} \beta^{2}\left|k_{0}-k_{1}\right|^{-2 \alpha d} \int_{0}^{1} d s_{k_{0} k_{1}} \int_{0}^{s_{k_{0} k_{1}}} d s_{k_{0} k_{1}}^{\prime} E\left(\mathscr{J}_{k_{0} k_{1}}^{2}\left[\partial_{k_{0} k_{1}}^{2}\left(\mu_{\Lambda \varrho} \sigma_{A}-\mu_{\Lambda \underline{\varrho}^{\prime}} \sigma_{A}\right)^{2}\right]_{C}\right. \\
& \leqq \sum_{k_{1} \in \Lambda \backslash k_{0}} \beta^{2} D_{\beta}\left|k_{0}-k_{1}\right|^{-2 \alpha d} E\left[\left(\mu_{\Lambda \varrho} \sigma_{A}-\mu_{\Lambda \underline{\varrho}^{\prime}} \sigma_{A}\right)^{2}\right. \\
& \left.+\left(\mu_{\Lambda \underline{Q}} \sigma_{A \backslash k_{0}} \sigma_{k_{1}}-\mu_{\Lambda \underline{Q}} \sigma_{A \backslash k_{0}} \sigma_{k_{1}}\right)^{2}+\left(\mu_{\Lambda \varrho} \sigma_{k_{0}} \sigma_{k_{1}}-\mu_{\Lambda \underline{Q}^{\prime}} \sigma_{k_{0}} \sigma_{k_{1}}\right)^{2}\right]_{C^{\prime}},
\end{aligned}
$$


where $C^{\prime}$ differs from $C$ by the additional condition that $K_{k_{0} k_{1}}=0$. Since there are three terms on the r.h.s. of (2.13), the constant $D_{\beta}$ is given by

$$
D_{\beta}=K_{1} \sum_{n=1}^{\infty} \frac{\left(3 K \beta^{2}\right)^{n-1}}{(2 n) !} E \mathscr{F}_{i j}^{2 n}
$$

for some constant $K_{1}>0$. The factor $[(2 n) !]^{-1}$ comes from the integrals

$$
\int_{0}^{1} d s_{k_{0} k_{1}} \int_{0}^{s_{k_{0} k_{1}}} \cdots \int_{0}^{s_{k_{0} k_{1}}^{(2 n-1)}} d s_{k_{0} k_{n}}^{(2 n)}=\frac{1}{(2 n) !} .
$$

Since we have assumed that

$$
E \mathscr{J}_{i j}^{2 n} \leqq \gamma^{2 n}(2 n) !,
$$

for some finite $\gamma>0$, the constant $D_{\beta}$ is finite, for $0 \leqq \beta<\beta_{0}$, if $\beta_{0}$ is small enough. Moreover, $D_{\beta}$ is obviously increasing in $\beta$, so the bound on $D_{\beta}$ is uniform on $\left[0, \beta_{0}-\delta\right]$, for any $\delta>0$.

Now, we observe that the terms under the expectation $E$ on the r.h.s. of (2.14) have the structure of

$$
\left.I_{B}\left(C^{\prime}\right) \equiv E\left(\mu_{\Lambda \underline{Q}} \sigma_{B}-\mu_{\Lambda \underline{Q}^{\prime}} \sigma_{B}\right)^{2}\right|_{C^{\prime}}
$$

where $C^{\prime}$ is the condition that $K_{k_{0} A^{c}}=0, K_{k_{0}}=0$, for all $i \leqq k_{1}$, and $B=A$, $\left(A \backslash k_{0}\right) \cup k_{1},\left\{k_{0}, k_{1}\right\}$. For the term $I_{A}\left(C^{\prime}\right)$ we can derive an identity analogous to (2.11) and bound the second term on the r.h.s. as in (2.14). (The circumstance that the condition $K_{k_{0} \Lambda^{c}}=0$ has been replaced by $C^{\prime}$ does not impede this procedure!) Thus, by iteration of (2.11) and (2.14) for $I_{A}\left(C^{\prime}\right)$, we find

$$
\begin{aligned}
& I_{A}\left(K_{k_{0} \Lambda^{c}}=0\right) \leqq\left(1+\beta^{2} \bar{D}_{\beta}\right) I_{A}\left(K_{k_{0} \Gamma}=0\right) \\
& +\left.\beta^{2} \bar{D}_{\beta} \sum_{k_{1} \in \Lambda \backslash A}\left|k_{0}-k_{1}\right|^{-2 \alpha d} E\left(\mu_{\Lambda \underline{\varrho}} \sigma_{A \backslash k_{0}} \sigma_{k_{1}}-\mu_{\Lambda \underline{Q}^{\prime}} \sigma_{A \backslash k_{0}} \sigma_{k_{1}}\right)^{2}\right|_{C^{\prime}} \\
& +\left.\beta^{2} \bar{D}_{\beta} \sum_{k_{1} \in A \backslash k_{0}}\left|k_{0}-k_{1}\right|^{-2 \alpha d} E\left(\mu_{\Lambda \varrho} \sigma_{A \backslash\left\{k_{0}, k_{1}\right\}}-\mu_{\Lambda \underline{Q}^{\prime}} \sigma_{A \backslash\left\{k_{0}, k_{1}\right\}}\right)^{2}\right|_{C^{\prime}} \\
& +\left.\beta^{2} \bar{D}_{\beta} \sum_{\substack{k_{1} \in \Lambda \\
k_{1} \neq k_{0}}}\left|k_{0}-k_{1}\right|^{-2 \alpha d} E\left(\mu_{\Lambda \varrho} \sigma_{k_{0}} \sigma_{k_{1}}-\mu_{\Lambda \underline{\varrho}^{\prime}} \sigma_{k_{0}} \sigma_{k_{1}}\right)^{2}\right|_{C^{\prime}} .
\end{aligned}
$$

Here, the constant $\bar{D}_{\beta}$ is bounded by

$$
\bar{D}_{\beta} \leqq \sum_{n=1}^{\infty}\left(\beta^{2} D_{\beta} \sum_{k \neq i}|k-i|^{-2 \alpha d}\right)^{n} .
$$

Hence $\bar{D}_{\beta}$ is finite, for $\alpha>\frac{1}{2}$ and $\beta$ small enough. It is increasing in $\beta$ on $\mathbb{R}^{+}$. In conclusion, $\bar{D}_{\beta}$ is uniformly bounded on $\left[0, \beta_{0}-\delta\right]$, for some $\beta_{0}>0$, for any $\delta>0$.

From (2.10) and (2.19) we conclude that

$$
\begin{aligned}
I_{A} \equiv & E\left(\mu_{A \underline{\underline{g}}} \sigma_{A}-\mu_{A \underline{g}^{\prime}} \sigma_{A}\right)^{2} \leqq a \beta^{2} \sum_{j \in A^{\prime} \backslash \Lambda}\left|k_{0}-j\right|^{-2 \alpha d}+\left(1+\beta^{2} \bar{D}_{\beta}\right) I_{A}\left(K_{k_{0} \Gamma}=0\right) \\
& +\beta^{2} \bar{D}_{\beta} \sum_{k_{1} \in \Lambda \backslash A}\left|k_{0}-k_{1}\right|^{-2 \alpha d} I_{\left(A \backslash k_{0}\right) \cup k_{1}}\left(C^{\prime}\right) \\
& +\beta^{2} \bar{D}_{\beta} \sum_{k_{1} \in A \backslash k_{0}}\left|k_{0}-k_{1}\right|^{-2 \alpha d} I_{A \backslash\left\{k_{0}, k_{1}\right\}}\left(C^{\prime}\right) \\
& +\beta^{2} \bar{D}_{\beta} \sum_{\substack{k_{1} \in A \\
k_{1} \neq k_{0}}}\left|k_{0}-k_{1}\right|^{-2 \alpha d} I_{\left\{k_{0}, k_{1}\right\}}\left(C^{\prime}\right) \\
&
\end{aligned}
$$


where, we recall, $C^{\prime}$ is the condition $\left\{K_{k_{0} \Lambda^{c}}=0, K_{k_{0} i}=0\right.$, for all $\left.i \leqq k_{1}\right\}$. We remark that if $d \varrho(\sigma)$ is symmetric in $\sigma$ then $I_{A}\left(K_{k_{0} \Gamma}=0, \widetilde{C}\right)=0$, for arbitrary $\widetilde{C}$.

Next, we notice that the term $I_{\left(A \backslash k_{0}\right) \cup k_{1}}\left(C^{\prime}\right), k_{1} \in \Lambda \backslash A$, has a form similar to the one of $I_{A}$, so that it can be bounded by an inequality analogous to (2.21). More precisely, we start our expansion of $I_{\left(A \backslash k_{0}\right) \cup k_{1}}$ at the point $k_{1}$ and obtain, after repeating the steps $(2.8)-(2.21)$,

where

$$
\begin{aligned}
I_{\left(A \backslash k_{0}\right) \cup k_{1}}\left(C^{\prime}\right) \leqq & a \beta^{2} \sum_{j \in \Lambda^{\prime} \backslash A}\left|k_{1}-j\right|^{-2 \alpha d}+\left(1+\beta^{2} \bar{D}_{\beta}\right) I_{\left(A \backslash k_{0}\right) \cup k_{1}}\left(C^{\prime}, K_{k_{1} \Gamma}=0\right) \\
& +\beta^{2} \bar{D}_{\beta} \sum_{\substack{k_{2} \in \Lambda \backslash A \\
k_{2} \neq k_{1}}}\left|k_{1}-k_{2}\right|^{-2 \alpha d} I_{\left(A \backslash k_{0}\right) \cup k_{2}}\left(C^{\prime \prime}\right) \\
& +\beta^{2} \bar{D}_{\beta} \sum_{\substack{k_{2} \in A \backslash k_{0} \\
k_{2} \neq k_{1}}}\left|k_{1}-k_{2}\right|^{-2 \alpha d} I_{A \backslash\left\{k_{0}, k_{2}\right\}}\left(C^{\prime \prime}\right) \\
& +\beta^{2} \bar{D}_{\beta} \sum_{\substack{k_{2} \in \Lambda \\
k_{2} \neq k_{1}, k_{0}}}\left|k_{1}-k_{2}\right|^{-2 \alpha d} I_{\left\{k_{1}, k_{2}\right\}}\left(C^{\prime \prime}\right)
\end{aligned}
$$

$$
C^{\prime \prime}=\left\{K_{k_{0}, \Lambda^{c}}=0, K_{k_{0} i}=0, i \leqq k_{1} ; K_{k_{1} \Lambda^{c}}=0, K_{k_{1} i^{\prime}}=0, i^{\prime} \leqq k_{2}\right\} .
$$

The order $\prec$ may be chosen to depend on $k_{1}$ in such a way that the elements $k_{2} \in A \backslash k_{0}$ are smaller, with respect to $\prec$, than the elements $k_{2} \in \Lambda \backslash A$.

Next, we may feed the bound (2.22) back into inequality (2.21), and iterate this step indefinitely. At the $n$-th step of our iteration we start from a term $I_{\left(A \backslash k_{0}\right) \cup k_{n-1}}\left(C^{(n-1)}\right)$, where

$$
C^{(n-1)}=\left\{K_{k_{m} \Lambda^{c}}=0, K_{k_{m} i}=0, \forall i \leqq k_{m+1}\right\}_{m=0}^{n-2},
$$

and we choose $\prec$ such that all sites $i \in A \backslash k_{0}$, with $i \leqq k_{m+1}, m=0, \ldots, n-2$, are smaller, with respect to $\prec$, than the remaining sites. At the $n$-th step we start our expansion steps at the site $k_{n-1}$. Let us summarize the main features of our iteration encountered at the $n$-th step:

a) $k_{n} \neq k_{n-2}$, since $K_{k_{n-2} k_{n-1}}$ has been set to zero at the $(n-1)$-st step. Hence $k_{n}$ $\neq k_{n-1}, k_{n-2}$.

b) If $k_{n-1}=k_{m}, m=0, \ldots, n-4$, then the term $\alpha \beta^{2} \sum_{j \in \Lambda^{\prime} \backslash \Lambda}\left|k_{n-1}-j\right|^{-2 \alpha d}$ in the expansion of $I_{\left(A \backslash k_{0}\right) \cup k_{n-1}}\left(C^{(n-1)}\right)$ is absent, since $K_{k_{m} \Lambda^{c}}$ has been set to zero at the $(m+1)$-st step.

c) If $k_{n}=k_{m}, m=0, \ldots, n-3$, and the interaction term $K_{k_{n-1} k_{m}}$ was the last coupling between $\sigma_{k_{m}}$ and $\sigma_{\Gamma \backslash k_{m}}$, then

$$
I_{\left(A \backslash k_{0}\right) \cup k_{n}}\left(C^{(n)}\right)=\bar{\varrho} I_{A \backslash k_{0}}\left(C^{(n)}\right),
$$

where

$$
\bar{\varrho} \equiv \varrho\left(\sigma_{k_{n}}\right)^{2}=\left(\int d \varrho(\sigma) \sigma\right)^{2},
$$

as follows directly from (2.2), (2.3). If $d \varrho$ is even in $\sigma$ then $I_{\left(A \backslash k_{0}\right) \cup k_{n}}\left(C^{(n)}\right)=0$. Similarly,

$$
\left.\begin{array}{l}
I_{A}\left(K_{k_{0} \Gamma}=0\right)=\bar{\varrho} I_{A \backslash k_{0}}\left(K_{k_{0} \Gamma}=0\right), \\
I_{\left(A \backslash k_{0}\right) \cup k_{n}}\left(C^{(n-1)}, K_{k_{n} \Gamma}=0\right)=\varrho I_{A \backslash k_{0}}\left(C^{(n-1)}, K_{k_{n} \Gamma}=0\right) .
\end{array}\right\}
$$


d) If $k_{n}=k_{m}, m=0, \ldots, n-3$, then the bond $\left(k_{n-1}, k_{n}\right) \neq\left(k_{l-1}, k_{l}\right)$, for all $l \leqq n-3$, because otherwise $K_{k_{n-1} k_{n}}$ would already have been set to zero at the $l$-th step.

e) At the $n$-th step we generate the following terms:

i) If $k_{n-1}$ was not met before a term

$$
a \beta^{2} \sum_{j \in \Lambda^{\prime} \backslash \Lambda}\left|k_{n-1}-j\right|^{-2 \alpha d} .
$$

ii) A term

$$
\left(1+\beta^{2} \bar{D}_{\beta}\right) \bar{\varrho} I_{A \backslash k_{0}}\left(C^{(n-1)}, K_{k_{n-1} \Gamma}=0\right)
$$

and we note that $\left|A \backslash k_{0}\right|=|A|-1<|A|$.

iii) Terms of the form

$$
\beta^{2} \bar{D}_{\beta} \sum_{k_{n} \neq k_{n-1}, k_{n-2}}\left|k_{n-1}-k_{n}\right|^{-2 \alpha d} I_{B}\left(C^{(n)}\right),
$$

where one term has $B=\left(A \backslash k_{0}\right) \cup k_{n}$, i.e. $|B|=|A|$, for which the iteration will continue, but the other terms have $|B|<|A|$.

Using (2.21) and (2.22) and iterating in the manner just described above, we finally get the bound

$$
\begin{aligned}
& I_{A} \leqq a \beta^{2} \sum_{j \in A^{\prime} \backslash \Lambda}\left\{\sum_{n=0}^{\infty}\left(\beta^{2} \bar{D}_{\beta}\right)^{n} \sum_{\substack{k_{m} \in \Lambda \backslash A \\
k_{m}+1 \neq k_{m}, k_{m-1} \\
k_{n} \neq k_{m}, m<n}}\left(\prod_{m=1}^{n}\left|k_{m-1}-k_{m}\right|^{-2 \alpha d}\right)\left|k_{n}-j\right|^{-2 \alpha d}\right\} \\
& +\left(1+\beta^{2} \bar{D}_{\beta}\right) \bar{\varrho} \sum_{n=0}^{\infty}\left(\beta^{2} \bar{D}_{\beta}\right)^{n-1} \\
& \times \sum_{\substack{k_{m} \in \Lambda \backslash A \\
k_{m}+1 \neq k_{m}, k_{m-1}}} \prod_{m=1}^{n-1}\left|k_{m-1}-k_{m}\right|^{-2 \alpha d} \cdot I_{A \backslash k_{0}}\left(C^{(n)}, K_{k_{n} \Gamma}=0\right)
\end{aligned}
$$

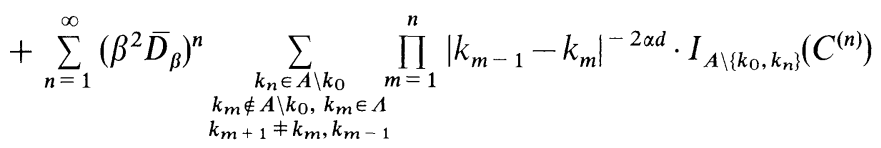

$$
\begin{aligned}
& +\sum_{n=1}^{\infty}\left(\beta^{2} \bar{D}_{\beta}\right)^{n} \sum_{\substack{k_{m} \in \Lambda \\
k_{m}+1 \neq k_{m}, k_{m-1}}} \prod_{m=1}^{n}\left|k_{m-1}-k_{m}\right|^{-2 \alpha d} I_{\left\{k_{n-1}, k_{n}\right\}}\left(C^{(n)}\right),
\end{aligned}
$$

with $C^{(n)}=\left\{K_{k_{m} \Lambda^{c}}=0, K_{k_{m} i}=0, \forall i \leqq k_{m+1}\right\}_{m=0}^{n-1}$. The first term on the r.h.s. of (2.27) can be estimated by

$$
a \beta^{2}\left\{\sum_{n=0}^{\infty}\left(\beta^{2} \bar{D}_{\beta} b\right)^{n}\right\} \sum_{j \in \Lambda^{\prime} \backslash \Lambda}\left|k_{0}-j\right|^{-2 \alpha d},
$$

which is finite if $\beta$ is sufficiently small. Here $b$ is a finite constant with the property that

$$
\sum_{\substack{k^{\prime} \in A \\ k^{\prime} \neq k}}\left|k-k^{\prime}\right|^{-2 \alpha d}\left|k^{\prime}-j\right|^{-2 \alpha d} \leqq b|k-j|^{-2 \alpha d}
$$


for $k \in \Lambda, j \in \Lambda^{\prime} \backslash \Lambda$, and it is assumed that $\alpha>\frac{1}{2}$. The series occurring in the last three terms on the r.h.s. of (2.27) may also be estimated using (2.29). For example,

$$
\begin{aligned}
& \sum_{n=1}^{\infty}\left(\beta^{2} \bar{D}_{\beta}\right)^{n} \sum_{\substack{k_{m} \in \Lambda \backslash A \\
k_{m+1} \neq k_{m}, k_{m-1}}} \prod_{m=1}^{n}\left|k_{m-1}-k_{m}\right|^{-2 \alpha d} \\
& \quad \leqq \beta^{2} \bar{D}_{\beta}\left(1-\beta^{2} D_{\beta} b\right)^{-1}
\end{aligned}
$$

which is finite for $\beta$ small enough.

Next, we note that the factors $I_{B}(\ldots), B=A \backslash k_{0}, A \backslash\left\{k_{0}, k_{n}\right\},\left\{k_{n-1}, k_{n}\right\}$, appearing in the last three terms on the r.h.s. of (2.27) have the pleasant feature that, for $|A|>2$,

$$
|B| \leqq|A|-1 .
$$

This offers the opportunity to induct in the cardinality of $|A|$. In order to complete a proof of Proposition 2.1 by induction in $|A|$ it remains to be shown that

and

$$
\lim _{\Lambda \nearrow \Gamma} E\left(\mu_{\Lambda \underline{\underline{g}}} \sigma_{i}-\mu_{\Lambda \underline{g}^{\prime}} \sigma_{i}\right)^{2}=0
$$

$$
\lim _{\Lambda \nearrow \Gamma} E\left(\mu_{\Lambda \varrho} \sigma_{i} \sigma_{j}-\mu_{\Lambda \varrho^{\prime}} \sigma_{i} \sigma_{j}\right)^{2}=0 .
$$

This is the content of the following lemma.

Lemma 2.3. If $0 \leqq \beta<\beta_{0}$, for some sufficiently small $\beta_{0}>0$, then

$$
\left.E\left(\mu_{\Lambda \underline{\underline{\varrho}}} \sigma_{i}-\mu_{\Lambda \underline{\varrho}^{\prime}} \sigma_{i}\right)^{2}\right|_{C} \leqq K^{\prime} \sum_{j \in \Lambda^{c}}|i-j|^{-2 \alpha d}
$$

and

$$
\left.E\left(\mu_{\Lambda \varrho} \sigma_{i} \sigma_{l}-\mu_{\Lambda \varrho^{\prime}} \sigma_{i} \sigma_{l}\right)^{2}\right|_{C} \leqq K^{\prime \prime} \sum_{k_{0}=i, l} \sum_{j \in \Lambda^{c}}\left|k_{0}-j\right|^{-2 \alpha d},
$$

where $K^{\prime}$ and $K^{\prime \prime}$ are finite constants which depend on $\beta$ but are independent of $\Lambda, \varrho$, $\varrho^{\prime}$ and the condition $C$ imposed on the l.h.s. of ( $i$ ) and (ii).

Proof. By Taylor's theorem with remainder

$$
\begin{aligned}
E\left(\mu_{\Lambda \varrho} \sigma_{i}-\mu_{\Lambda \varrho^{\prime}} \sigma_{i}\right)^{2}= & \left.E\left(\mu_{\Lambda \varrho} \sigma_{i}-\mu_{\Lambda \underline{\varrho}^{\prime}} \sigma_{i}\right)^{2}\right|_{K_{i} \Lambda^{c}=0}+\sum_{j \in \Lambda^{c}} \beta^{2}|i-j|^{-2 \alpha d} \\
& \times \int_{0}^{1} d s_{i j} \int_{0}^{s_{i j}} d s_{i j}^{\prime} E\left(\left.\mathscr{J}_{i j}^{2} \partial_{i j}^{2}\left(\mu_{\Lambda \varrho} \sigma_{i}-\mu_{\Lambda \underline{\varrho}^{\prime}} \sigma_{i}\right)^{2}\right|_{C}\right),
\end{aligned}
$$

where $C$ is the condition $\left\{K_{i l}=0, \forall l \prec j, S_{i j}^{\prime}\right\}$. The integrand on the r.h.s. of (2.34) is obviously bounded, so we obtain

$$
I_{i} \equiv E\left(\mu_{\Lambda \varrho} \sigma_{i}-\mu_{\Lambda \varrho^{\prime}} \sigma_{i}\right)^{2} \leqq I_{i}\left(K_{i \Lambda^{c}}=0\right)+a \beta^{2} \sum_{j \in \Lambda^{c}}|i-j|^{-2 \alpha d},
$$

for some finite constant $a$ independent of $\beta$ and $\Lambda$.

Next, we expand $I_{i}\left(K_{i \Lambda^{c}}=0\right)$ in $J_{i k}, k \in \Lambda$, to second order and obtain

$$
\begin{aligned}
I_{i}\left(K_{i \Lambda^{c}}=0\right)= & I_{i}\left(K_{i \Lambda^{c} \cup k}=0\right)+\beta^{2}|i-k|^{-2 \alpha d} \\
& \times \int_{0}^{1} d s_{i k} \int_{0}^{s_{i k}^{\prime}} d s_{i k}^{\prime} E\left(\left.\mathscr{J}_{i k}^{2} \partial_{i j}^{2}\left(\mu_{\Lambda \varrho} \sigma_{i}-\mu_{\Lambda \varrho^{\prime}} \sigma_{i}\right)^{2}\right|_{C}\right),
\end{aligned}
$$


where $C$ is the condition $\left\{K_{i \Lambda^{c}}=0, S_{i k}^{\prime}\right\}$. We now use (2.13) to bound the integrand on the r.h.s. of (2.36). This yields

$$
I_{i}\left(K_{i \Lambda^{c}}=0\right) \leqq I_{i}\left(K_{i \Lambda^{c} \cup k}=0\right)+\beta^{2}|i-k|^{-2 \alpha d} \gamma K\left[I_{i}(C)+I_{k}(C)+I_{\{i, k\}}(C)\right] .
$$

The term $I_{\{i, k\}}(C)=\left.E\left(\mu_{A \varrho} \sigma_{i} \sigma_{k}-\mu_{A \rho^{\prime}} \sigma_{i} \sigma_{k}\right)^{2}\right|_{C}$ will be estimated in the proof of part (ii) of Lemma 2.3. All three terms, $I_{i}(C), I_{k}(C)$, and $I_{\{i, k\}}(C)$, are now expanded to second order in $S_{i k}^{\prime}$ again, whereupon we apply (2.13) another time. After repeating this procedure indefinitely we obtain

$$
I_{i}\left(K_{i \Lambda^{c}}=0\right) \leqq I_{i}\left(K_{i \Lambda^{c} \cup k}=0\right)+\beta^{2} D_{\beta}|i-k|^{-2 \alpha d}\left[I_{i}\left(C^{\prime}\right)+I_{k}\left(C^{\prime}\right)+I_{\{i, k\}}\left(C^{\prime}\right)\right],
$$

where $D_{\beta}$ is given in (2.15); see (2.14).

Applying an inequality analogous to $(2.38)$ to $I_{i}\left(K_{i \Lambda^{c} \cup k}=0\right)$, for some $k^{\prime}>k$, etc. we finally get

$$
I_{i}\left(K_{i \Lambda^{c}}=0\right) \leqq I_{i}\left(K_{i \Gamma}=0\right)+\beta^{2} D_{\beta} \sum_{\substack{k \in A \\ k \neq i}}|i-k|^{-2 \alpha d}\left[I_{i}\left(C_{k}\right)+I_{k}\left(C_{k}\right)+I_{\{i, k\}}\left(C_{k}\right)\right],
$$

where $C_{k}$ corresponds to $\left\{K_{i \Lambda^{c}}=0, K_{i k^{\prime}}=0, \forall k^{\prime} \leqq k\right\}$.

Now we note that

$$
I_{i}\left(K_{i \Gamma}=0\right)=\left.E\left(\mu_{\Lambda \underline{\underline{a}} \sigma_{i}}-\mu_{\Lambda \underline{\underline{Q}}} \sigma_{i}\right)^{2}\right|_{K_{i \Gamma}=0}=0 .
$$

Next, we apply an analogue of (2.39) to the term $I_{i}\left(C_{k}\right)$ on the r.h.s. of (2.39), use (2.40) again and iterate. As in (2.19) and (2.20) we find

$$
I_{i}\left(K_{i \Lambda^{c}}=0\right) \leqq \beta^{2} \bar{D}_{\beta} \sum_{\substack{k \in A \\ k \neq i}}|i-k|^{-2 \alpha d}\left[I_{k}\left(C_{k}\right)+I_{\{i, k\}}\left(C_{k}\right)\right] .
$$

Combining (2.35) and (2.41) we obtain the bound

$$
I_{i} \leqq a \beta^{2} \sum_{j \in A^{c}}|i-j|^{-2 \alpha d}+\beta^{2} \bar{D}_{\beta} \sum_{\substack{k \in A \\ k \neq i}}|i-k|^{-2 \alpha d} \cdot\left[I_{k}\left(C_{k}\right)+I_{\{i, k\}}\left(C_{k}\right)\right] .
$$

The last term on the r.h.s. of (2.42) is bounded in part (ii) of Lemma 2.3. For the term proportional to $I_{k}\left(C_{k}\right)$ an inequality analogous to (2.42), with $i$ replaced by $k$, provides an upper bound, and this can be iterated indefinitely. We find

$$
\begin{aligned}
I_{i} \leqq & \sum_{n=0}^{\infty}\left(\beta^{2} \bar{D}_{\beta}\right)^{n} \sum_{\substack{k_{1}, \ldots, k_{n} \in \Lambda i i \\
k_{i+2} \neq k_{i+i}, k_{i}}}\left|i-k_{1}\right|^{-2 \alpha d} \ldots\left|k_{n-1}-k_{n}\right|^{-2 \alpha d} \\
& \times\left\{\sum_{j \in \Lambda^{c}} a \beta^{2}\left|k_{n}-j\right|^{-2 \alpha d}+I_{\left\{k_{n-1}, k_{n}\right\}}\left(C^{(n)}\right)\right\}
\end{aligned}
$$

where $C^{(n)}$ is a condition depending on $\left\{i, k_{1}, \ldots, k_{n}\right\}, I_{\left\{k_{-1}, k_{0}\right\}} \equiv 0$, and $I_{\left\{k_{0}, k_{1}\right\}} \equiv I_{\left\{i, k_{1}\right\}}$. The proof of part (i) is completed by using (2.29) and part (ii).

We now turn to the proof of part (ii). As in (2.10) we find by removing $J_{i j}$ and $J_{l j}$, $j \in \Lambda^{c}$,

$$
\begin{aligned}
\left.E\left(\mu_{\Lambda \underline{\underline{Q}}} \sigma_{i} \sigma_{l}-\mu_{\Lambda \underline{Q}} \sigma_{i} \sigma_{l}\right)^{2}\right|_{C} \leqq & I_{\{i, l, l}\left(C, K_{\{i, l\}, \Lambda^{c}}=0\right) \\
& +a \beta^{2} \sum_{j \in \Lambda^{c}}\left(|i-j|^{-2 \alpha d}+|l-j|^{-2 \alpha d}\right) .
\end{aligned}
$$


Next, we perform an expansion step in $J_{i k}, k \in \Lambda \backslash i$, using (2.11), and iterate this step using (2.13), indefinitely, until we obtain a bound analogous to (2.19). Note that on the r.h.s. of (2.19) the term proportional to $\left.E\left(\mu_{\Lambda \varrho} \sigma_{A \backslash k_{0}} \sigma_{k_{1}}-\mu_{\Lambda \varrho} \sigma_{A \backslash k_{0}} \sigma_{k_{1}}\right)^{2}\right|_{C^{\prime}}$ is absent, since $\sigma_{A \backslash k_{0}} \sigma_{k_{1}}=1$, for $k_{1} \in A \backslash k_{0}, k_{0}=i$. Hence

$$
\begin{aligned}
I_{\{i, l\}}\left(K_{\{i, l\} \Lambda^{c}}=0\right) \leqq & \left(1+\beta^{2} \bar{D}_{\beta}\right) I_{\{i, l\}}\left(K_{i \Gamma}=0\right)+\beta^{2} \bar{D}_{\beta}|i-l|^{-2 \alpha d} I_{\{i, l\}}\left(C_{1}\right) \\
& +\beta^{2} \bar{D}_{\beta} \sum_{k_{1} \in \Lambda \backslash\{i, l\}}\left|i-k_{1}\right|^{-2 \alpha d} \cdot\left\{I_{\left\{l, k_{1}\right\}}\left(C_{2}\right)+I_{\left\{i, k_{1}\right\}}\left(C_{2}\right)\right\},
\end{aligned}
$$

where $C_{1}$ corresponds to $\left\{K_{\{i, l\} \Lambda^{c}}=0, K_{i l}=0\right\}$ and $C_{2}$ to $\left\{K_{\{i, l\} \Lambda^{c}}=0, K_{i m}=0\right.$, $\left.\forall m \leqq k_{1}\right\}$. Now we note that

$$
I_{\{i, l\}}\left(K_{i \Gamma}=0\right)=\varrho I_{l}\left(K_{i \Gamma}=0\right),
$$

where, we recall, $\bar{\varrho}=\left(\int \sigma_{i} d \varrho\left(\sigma_{i}\right)\right)^{2}$. We may and do assume that the order $\prec$ is chosen such that if $k_{1} \in \Lambda \backslash\{i, l\}$ then $k_{1}>l$, and hence $K_{i l}=0$. The second term on the r.h.s. of $(2.45)$ has the same structure as $I_{\{i, l\}}\left(K_{\{i, l\} \Lambda^{c}}=0\right)$, hence $(2.45)$ can be iterated.

This yields the bound

$$
\begin{aligned}
I_{\{i, l\}}\left(K_{\{i, l\} \Lambda^{c}}=0\right) \leqq & \beta^{2} F_{\beta}\left(1+\beta^{2} \bar{D}_{\beta}\right) \bar{\varrho} I_{l}\left(K_{i \Gamma}=0\right)+\beta^{2} G_{\beta} \\
& \times \sum_{k_{1} \in \Lambda \backslash\{i, l\}}\left|i-k_{1}\right|^{-2 \alpha d}\left\{I_{\left\{i, k_{1}\right\}}(C)+I_{\left\{l, k_{1}\right\}}(C)\right\},
\end{aligned}
$$

where $F_{\beta}$ and $G_{\beta}$ are constants which are uniformly bounded in $\beta \in\left[0, \beta_{0}-\delta\right]$, for any $\delta>0$, if $\beta_{0}$ is chosen small enough. Moreover, $C$ corresponds to $\left\{K_{\{i, l\} A^{c}}=0\right.$, $\left.K_{i l}=0, K_{i m}=0, \forall m \leqq k_{1}\right\}$. The point is now that we may plug inequality (2.43) into (2.47) by replacing the first term on the r.h.s. of (2.47) by the r.h.s. of (2.43). Then we arrive at an inequality for the two-spin correlation $I_{\{i, l\}}(C)$ which can be iterated indefinitely. More precisely,

$$
\begin{aligned}
I_{\{i, l\}}(\tilde{C}) \leqq & a \beta^{2} \sum_{j \in \Lambda^{c}}\left(|i-j|^{-2 \alpha d}+|l-j|^{-2 \alpha d}\right) \\
& +\beta G_{\beta} \sum_{k_{1} \in \Lambda \backslash\{i, l\}}\left|i-k_{1}\right|^{-2 \alpha d}\left\{I_{\left\{i, k_{1}\right\}}(\tilde{C}, C)+I_{\left\{l, k_{1}\right\}}(\tilde{C}, C)\right\} \\
& +\beta^{2} F_{\beta}\left(1+\beta^{2} \bar{D}_{\beta}\right) \beta^{2} L_{\beta} \sum_{j \in \Lambda^{c}}|i-j|^{-2 \alpha d} \\
& +\beta^{2} F_{\beta}\left(1+\beta^{2} \bar{D}_{\beta}\right)\left\{\sum_{n=1}^{\infty}\left(\beta^{2} \bar{D}_{\beta}\right)^{n} \sum_{\substack{k_{1}, \ldots, k_{n} \in \Lambda \backslash i \\
k_{i}+2 \neq k_{i+1}, k_{i}}}\left|i-k_{1}\right|^{-2 \alpha d} \ldots\right. \\
& \left.\ldots\left|k_{n-1}-k_{n}\right|^{-2 \alpha d} I_{\left\{k_{n-1}, k_{n}\right\}}\left(\tilde{C}, K_{i \Gamma}=0, C^{(n)}\right)\right\}
\end{aligned}
$$

where $L_{\beta}$ is a finite constant, for $0 \leqq \beta<\beta_{0}, \beta_{0}$ small enough; moreover, $I_{\left\{k_{0}, k_{1}\right\}} \equiv I_{\left\{i, k_{1}\right\}}$. The condition $C^{(n)}$ has been described after (2.27). Using (2.29), we see that iteration of (2.48) yields an upper bound for $I_{\{i, l\}}(\widetilde{C})$ which is finite and tends to 0 , as $\Lambda \nearrow \Gamma$, provided $0 \leqq \beta<\beta_{0}$, with $\beta_{0}$ small enough. This completes the proof of Lemma 2.3, and hence Proposition 2.1 is now proven, too. 


\subsection{Cluster Properties of the State $\mu_{\Lambda}$}

The main result of the present subsection is the following

Proposition 2.4. For $\alpha>\frac{1}{2}$ and $E$ as in (1.3), (1.4), there is a constant $\beta_{0}>0$ such that, for $0<\beta<\beta_{0}$ and arbitrary bounded subsets $A$ and $B$ of the lattice $\Gamma$,

$$
E\left(\mu_{\Lambda}\left(\sigma_{A} ; \sigma_{B}\right)^{2}\right) \leqq \beta^{2} \bar{C}_{\beta} \xi_{\beta}^{|A|}|B| d(A, B)^{-2 \alpha d},
$$

for $|A| \leqq|B|$, where $\xi_{\beta}$ and $\bar{C}_{\beta}$ are finite constants independent of $A, B$, and $A$, and $d(A, B)$ is the Euclidean distance between the sets $A$ and $B$.

Remarks. (1) After passing to the thermodynamic limit $\Lambda \nearrow \Gamma$, whose existence has been established in Sect. 2.1, we can extend Proposition 2.4 to the situation, where $A$ is a bounded set, but $B$ is unbounded (e.g. a cone). Since, for $\alpha>\frac{1}{2},|i-j|^{-2 \alpha d}$ is summable in $j$, we get clustering with $d(A, B)^{-2 \alpha d+d}$ which still tends to 0 , as $d(A, B) \rightarrow \infty$. (2) We also remark that when $A=\{i\}, B=\{j\}$ we can prove a lower bound on $E\left(\mu\left(\sigma_{i} ; \sigma_{j}\right)^{2}\right)$ which has the same decay as the upper bound [see Theorem 2, (1.19)].

Proof of Proposition 2.4. We assume that $|A| \leqq|B|$ and choose a site $k_{0} \in A$. We then expand in the couplings $J_{k_{0} j}, j \in B$, to second order. This yields

$$
\begin{aligned}
I_{A, B}= & I_{A, B}\left(K_{k_{0} B}=0\right)+\beta^{2} \sum_{j \in B}\left|k_{0}-j\right|^{-2 \alpha d} \\
& \times \int_{0}^{1} d s_{k_{0} j} \int_{0}^{s_{k_{0} J}} d s_{k_{0} j}^{\prime} E\left(\mathscr{J}_{k_{0} j}^{2}\left[\partial_{k_{0} j}^{2} \mu_{\Lambda}\left(\sigma_{A} ; \sigma_{B}\right)^{2}\right]_{S_{k_{0} J}^{k_{0} i}=0, i \prec j}\right),
\end{aligned}
$$

where

$$
\left.I_{A, B}(C) \equiv \mu_{\Lambda}\left(\sigma_{A} ; \sigma_{B}\right)^{2}\right|_{C}
$$

where $C$ is an arbitrary condition imposed on the couplings $J_{i j}, i, j \in \Lambda$, and $I_{A, B} \equiv I_{A, B}(\emptyset)$. The second term on the r.h.s. of (2.50) is clearly bounded by

$$
a \beta^{2} \sum_{j \in B}\left|k_{0}-j\right|^{-2 \alpha d}
$$

for some finite constant $a$ independent of $A, B, \Lambda$, and $\beta$. Next, we expand $I_{A, B}\left(K_{k_{0} B}=0\right)$ to second order in $J_{k_{0} k_{1}, c_{1}}$, for $k_{1} \in \Lambda \backslash B$. This yields

$$
\begin{aligned}
I_{A, B}\left(K_{k_{0} B}=0\right)= & \bar{\varrho} I_{A \backslash k_{0}, B}\left(K_{k_{0} \Gamma}=0\right)+\beta^{2} \sum_{\substack{k_{1} \in A \backslash B \\
k_{1} \neq k_{0}}}\left|k_{0}-k_{1}\right|^{-2 \alpha d} \\
& \left.\times \int_{0}^{1} d s_{k_{0} k_{1}} \cdot \int_{0}^{s_{k_{0} k_{1}}} d s_{k_{0} k_{1}}^{\prime} E\left(\mathscr{J}_{k_{0} k_{1}}^{2} \mid \partial_{k_{0} k_{1}}^{2} \mu_{\Lambda}\left(\sigma_{A} ; \sigma_{B}\right)^{2}\right]_{C}\right),
\end{aligned}
$$

where $C$ is the condition $\left\{K_{k_{0} B}=0, K_{k_{0} i}=0, \forall i \prec k_{1}, S_{k_{0} k_{1}}^{\prime}\right\}$. The second term on the r.h.s. of (2.53) is bounded with the help of the following inequality proven in Appendix A.1:

$$
\left|\left[\partial_{k_{0} k_{1}}^{2} \mu_{\Lambda}\left(\sigma_{B_{1}} ; \sigma_{B_{2}}\right)^{2}\right]_{C}\right| \leqq K^{\prime}\left\{I_{B_{1}, B_{2}}+I_{B_{1} \cup\left\{k_{0}, k_{1}\right\}, B_{2}}(C)+I_{\left\{k_{0}, k_{1}\right\}, B_{2}}(C)\right\},
$$

for some constant $K^{\prime}>0$ which is independent of $B_{1}, B_{2}$, and $C$. We now insert the r.h.s. of (2.54) on the r.h.s. of (2.53). Subsequently, we expand the resulting terms once again to second order in $S_{k_{0} k_{1}}^{\prime}$ and use (2.54) to bound the remainder term. 
Iterating this procedure indefinitely, we find

$$
\begin{aligned}
I_{A, B}\left(K_{k_{0} B}=0\right)= & \bar{\varrho} I_{A \backslash k_{0}, B}\left(K_{k_{0} \Gamma}=0\right)+\beta^{2} D_{\beta} \sum_{\substack{k_{1} \in \Lambda \backslash B \\
k_{1} \neq k_{0}}}\left|k_{0}-k_{1}\right|^{-2 \alpha d} \\
& \times\left\{I_{A, B}(C)+I_{\left(A \backslash k_{0}\right) \cup k_{1}, B}(C)+I_{\left\{k_{0}, k_{1}\right\}, B}(C)\right\},
\end{aligned}
$$

where $C$ corresponds to $\left\{K_{k_{0} B}=0, K_{k_{0} i}=0, \forall i \leqq k_{1}\right\}$, and

$$
D_{\beta}=K^{\prime \prime} \sum_{n=1}^{\infty} \frac{\left(3 K \beta^{2}\right)^{n-1}}{(2 n) !} E \mathscr{J}_{i j}^{2 n} \text {, }
$$

which is finite for $0 \leqq \beta<\beta_{0}$ if $\beta_{0}$ is small enough. [Recall that $E \mathscr{J}_{i j}^{2 n} \leqq \gamma^{2 n}(2 n)$ !, for all $n$, for some finite $\gamma$.] The factor $I_{A, B}(C)$ on the r.h.s. of $(2.55)$ has the same form as the 1.h.s. of (2.55). This permits us to iterate (2.55), and we get:

where

$$
\begin{aligned}
I_{A, B}\left(K_{k_{0} B}=0\right) \leqq & \left(1+\beta^{2} \bar{D}_{\beta}\right) \bar{\varrho} I_{A \backslash k_{0}, B}\left(K_{k_{0} \Gamma}=0\right) \\
& +\beta^{2} \bar{D}_{\beta} \sum_{\substack{k_{1} \in \Lambda \backslash B \\
k_{1} \neq k_{0}}}\left|k_{0}-k_{1}\right|^{-2 \alpha d}\left\{I_{\left(A \backslash k_{0}\right) \cup k_{1}, B}(C)+I_{\left\{k_{0}, k_{1}\right\}, B}(C)\right\},
\end{aligned}
$$

$$
\bar{D}_{\beta} \equiv D_{\beta} \sum_{n=0}^{\infty}\left(\beta^{2} D_{\beta} c\right)^{n}
$$

with

$$
c \equiv \sum_{\substack{j \in \Gamma \\ j \neq i}}|i-j|^{-2 \alpha d} .
$$

The constant $\bar{D}_{\beta}$ is bounded uniformly on compact subsets of $\left[0, \beta_{0}\right)$, for $\beta_{0}$ small enough. Hence, collecting (2.50), (2.52), and (2.57), we arrive at the inequality

$$
\begin{aligned}
I_{A, B} \leqq & a \beta^{2} \sum_{j \in B}\left|k_{0}-j\right|^{-2 \alpha d}+\left(1+\beta^{2} \bar{D}_{\beta}\right) \bar{\varrho} I_{A \backslash k_{0}, B}\left(K_{k_{0} \Gamma}=0\right) \\
& +\beta^{2} \bar{D}_{\beta} \sum_{k_{1} \in A \backslash B, k_{1} \notin A}\left|k_{0}-k_{1}\right|^{-2 \alpha d} I_{\left(A \backslash k_{0}\right) \cup k_{1}, B}(C) \\
& +\beta^{2} \bar{D}_{\beta} \sum_{k_{1} \in A \backslash k_{0}}\left|k_{0}-k_{1}\right|^{-2 \alpha d} I_{A \backslash\left\{k_{0}, k_{1}\right\}, B}(C) \\
& +\beta^{2} \bar{D}_{\beta} \sum_{\substack{k_{1} \in \Lambda \backslash B \\
k_{1} \neq k_{0}}}\left|k_{0}-k_{1}\right|^{-2 \alpha d} I_{\left\{k_{0}, k_{1}\right\}, B}(C),
\end{aligned}
$$

with $C$ given by $\left\{K_{k_{0} B}=0 ; K_{k_{0} i}=0, \forall i \leqq k_{1}\right\}$. We may require that $\prec$ has the property that if $k_{1} \in \Lambda \backslash(A \cup B)$ then $k_{1}>i$, for all $i \in A \backslash k_{0}$. We note that the factor $I_{\left(A \backslash k_{0}\right) \cup k_{1}, B}(C)$ in the third term on the r.h.s. of (2.59) has the same structure as $I_{A, B}$, in particular $\left|\left(A \backslash k_{0}\right) \cup k_{1}\right|=|A|$, and hence it obeys an upper bound analogous to (2.59). The remaining terms proportional to $I_{A^{\prime}, B}(\widetilde{C})$ on the r.h.s. of (2.59) have the feature that $\left|A^{\prime}\right|<|A|$, if $|A|>2$. Therefore, we may iterate (2.59) in $I_{\left(A \backslash k_{0}\right) \cup k_{1}, B}(C)$, 
starting at the site $k_{1}$. After infinitely many iterations we obtain the bound

$$
\begin{aligned}
& I_{A, B} \leqq a \beta^{2} \sum_{j \in B} \sum_{n=0}^{\infty}\left(\beta^{2} \bar{D}_{\beta}\right)^{n} \sum_{\substack{\left.k_{m} \in A \mid\left((A) k_{0}\right) \cup B\right) \\
k_{m+1}+1 \neq k_{m}, k_{m-1}-1 \\
k_{n} \neq k_{m}, m<n}}\left(\prod_{m=1}^{n}\left|k_{m-1}-k_{m}\right|^{-2 \alpha d}\right)\left|k_{n}-j\right|^{-2 \alpha d} \\
& +\left(1+\beta^{2} \bar{D}_{\beta}\right) \bar{\varrho} \sum_{n=0}^{\infty}\left(\beta^{2} \bar{D}_{\beta}\right)^{n} \\
& \times \sum_{k_{m} \in A \backslash\left(\left(A \backslash k_{0}\right) \cup B\right)}\left(\prod_{m=1}^{n}\left|k_{m-1}-k_{m}\right|^{-2 \alpha d}\right) I_{A \backslash k_{0}, B}\left(\widetilde{C}^{(n)}\right) \\
& +\sum_{n=1}^{\infty}\left(\beta^{2} \bar{D}_{\beta}\right)^{n} \sum_{k_{m} \in A \backslash\left(\left(A \backslash k_{0}\right) \cup B\right)}\left(\prod_{m=1}^{n}\left|k_{m-1}-k_{m}\right|^{-2 \alpha d}\right) I_{A \backslash\left\{k_{0}, k_{n}\right\}, B}\left(C^{(n)}\right) \\
& k_{n} \in \ddot{A} \backslash k_{0} \\
& +\sum_{n=1}^{\infty}\left(\beta^{2} \bar{D}_{\beta}\right)^{n} \sum_{\substack{k_{m} \in \Lambda \backslash B \\
k_{m+1} \neq k_{m}, k_{m-1}}}\left(\prod_{m=1}^{n}\left|k_{m-1}-k_{m}\right|^{-2 \alpha d}\right) I_{\left\{k_{n-1}, k_{n}\right\}, B}\left(C^{(n)}\right),
\end{aligned}
$$

where $C^{(n)}$ corresponds to $\left\{K_{k_{m} B}=0 ; K_{k_{m}, i}=0, i \leqq k_{m+1}\right\}_{m=0}^{n-1}$, and $\tilde{C}^{(n)}$ to $\left\{C^{(n)}, K_{k_{n} \Gamma}=0\right\}$. The first term on the r.h.s. of (2.60) is bounded by

$$
a \beta^{2} \sum_{n=0}^{\infty}\left(\beta^{2} \bar{D}_{\beta} b\right)^{n} \sum_{j \in B}\left|k_{0}-j\right|^{-2 \alpha d},
$$

provided $0 \leqq \beta<\beta_{0}$, for some sufficiently small $\beta_{0}$. The remaining terms on the r.h.s. are proportional to $I_{A^{\prime}, B}(\widetilde{C})$, with $\left|A^{\prime}\right|=|A|-1,\left|A^{\prime}\right|=|A|-2$, or $\left|A^{\prime}\right|=2$. The prefactors can be bounded by using

$$
\begin{aligned}
& \sum_{n=1}^{\infty} \beta^{2} \bar{D}_{\beta} \sum_{\begin{array}{c}
\left.k_{m} \in A \backslash\left((A) \mid k_{0}\right) \cup B\right) \\
k_{m+1} \neq k_{m}, k_{m-1}
\end{array}}\left(\prod_{m=1}^{n-1}\left|k_{m}-k_{m-1}\right|^{-2 \alpha d}\right)\left|k_{n-1}-k\right|^{-2 \alpha d} \\
& \leqq \beta^{2} F_{\beta}\left|k_{0}-k\right|^{-2 \alpha d} \text {, }
\end{aligned}
$$

for some constant $F_{\beta}$ which is uniformly bounded on compact subsets of $\left[0, \beta_{0}\right)$ if $\beta_{0}$ is small enough. Hence the proof of Proposition 2.4 can be completed by induction in $|A|$, provided we can prove it for $|A|=1$ and $|A|=2$. This is accomplished in the remainder of this section.

Lemma 2.5. For $\alpha>\frac{1}{2}, 0 \leqq \beta<\beta_{0}$, with $\beta_{0}$ small enough, there exist finite constants $C_{\beta}^{\prime}$ and $C_{\beta}^{\prime \prime}$ such that

(i) $\left.E \mu_{\Lambda}\left(\sigma_{i} ; \sigma_{B}\right)^{2}\right|_{C} \leqq C_{\beta}^{\prime} \sum_{j \in B}|i-j|^{-2 \alpha d}$, and

(ii) $\left.E \mu_{A}\left(\sigma_{i} \sigma_{l} ; \sigma_{B}\right)^{2}\right|_{C} \leqq C_{\beta}^{\prime \prime} \sum_{k=i, l} \sum_{j \in B}|k-j|^{-2 \alpha d}$.

The constants $C_{\beta}^{\prime}$ and $C_{\beta}^{\prime \prime}$ are independent of $A$ and $B$.

Proof. We start the proof of (i) with an expansion step, as in (2.50). Hence

$$
\begin{aligned}
I_{i, B}= & I_{i, B}\left(K_{i B}=0\right)+\beta^{2} \sum_{j \in B}|i-j|^{-2 \alpha d} \\
& \times \int_{0}^{1} d s_{i j} \int_{0}^{s_{i j}} d s_{i j}^{\prime} E\left(\mathscr{J}_{i j}^{2}\left[\partial_{i j}^{2} \mu_{A}\left(\sigma_{i} ; \sigma_{B}\right)^{2}\right]_{S_{i j}=0, l<j}\right) .
\end{aligned}
$$


The second term on the r.h.s. of (2.63) is bonded as in (2.52). Through steps, as in (2.53)-(2.58), we obtain

$$
\begin{aligned}
I_{i, B} \leqq & a \beta^{2} \sum_{j \in B}|i-j|^{-2 \alpha d}+\left(1+\beta^{2} \bar{D}_{\beta}\right) \varrho I_{\emptyset, B}\left(K_{i \Gamma}=0\right) \\
& +\beta^{2} \bar{D}_{\beta} \sum_{\substack{k \in \Lambda \backslash B \\
k \neq i}}|i-k|^{-2 \alpha d} I_{k, B}(C) \\
& +\beta^{2} \bar{D}_{\beta} \sum_{\substack{k \in \Lambda \backslash B \\
k \neq i}}|k-i|^{-2 \alpha d} I_{\{i, k\}, B}(C)
\end{aligned}
$$

where $C$ is given by $\left\{K_{i B}=0, K_{i l}=0, \forall l \leqq k\right\}$; see (2.59). From the definition of $I_{A, B}$ we see that

$$
I_{\emptyset, B}\left(K_{i \Gamma}=0\right)=0 \text {. }
$$

Since $I_{k, B}(C)$ has the same structure as $I_{i, B}$, we can iterate (2.64). This yields, using (2.29),

$$
\begin{aligned}
I_{i, B} \leqq & a \beta^{2}\left(\sum_{n=0}^{\infty}\left(\beta^{2} \bar{D}_{\beta} b\right)^{n}\right) \sum_{j \in B}|i-j|^{-2 \alpha d}+\sum_{n=1}^{\infty}\left(\beta^{2} \bar{D}_{\beta}\right)^{n} \sum_{\substack{k_{1}, \ldots, k_{n} \in \Lambda \backslash B \\
k_{i+1} \neq k_{i}}}\left|k_{0}-k_{1}\right|^{-2 \alpha d} \ldots \\
& \ldots\left|k_{n-1}-k_{n}\right|^{-2 \alpha d} I_{\left\{k_{n-1}, k_{n}\right\}, B}\left(C^{(n)}\right)
\end{aligned}
$$

where $k_{0} \equiv i$ and $C^{(n)}$ corresponds to

$$
\left\{K_{k_{m} B}=0, K_{k_{m} l}=0, \forall l \leqq k_{m+1}\right\}_{m=0}^{n-1} .
$$

Thus, in order to complete the proof of Lemma 2.5(i) we now must turn to the proof of (ii). We start as in (2.50), (2.63), i.e. we use the bound

$$
I_{\{i, l\}, B} \leqq I_{\{i, l\}, B}\left(K_{\{i, l\}, B}=0\right)+a \beta^{2} \sum_{k=i, l} \sum_{j \in B}|k-j|^{-2 \alpha d} .
$$

To bound the first term on the r.h.s. of (2.68), we proceed as in (2.53)-(2.58), expanding couplings between $i$ and $\Lambda \backslash B$ and choosing the order $\prec$ such that $l$ is the smallest site above $i$. This yields

$$
\begin{aligned}
I_{\{i, l\}, B}\left(K_{\{i, l\}, B}=0\right) \leqq & \left(1+\beta^{2} \bar{D}_{\beta}\right) \bar{\varrho} I_{l, B}\left(K_{i \Gamma}=0\right) \\
& +\beta^{2} \bar{D}_{\beta} \sum_{\substack{k_{1} \in \Lambda \backslash B \\
k_{1} \neq l}}\left|i-k_{1}\right|^{-2 \alpha d}\left\{I_{\left\{i, k_{1}\right\}, B}(C)+I_{\left\{l, k_{1}\right\}, B}(C)\right\} \\
& +\beta^{2} \bar{D}_{\beta}|i-l|^{-2 \alpha d} I_{\{i, l\}, B}(C)
\end{aligned}
$$

see (2.57). Since the last term on the r.h.s. of (2.69) has the same structure as the 1.h.s. of (2.69) we obtain by iterating (2.69) and inserting the result into (2.68),

$$
\begin{aligned}
& I_{\{i, l\}, B} \leqq a \beta^{2} \sum_{k=i, l} \sum_{j \in B}|k-j|^{-2 \alpha d}+\left(1+\beta^{2} \bar{D}_{\beta}\right) \varrho I_{l, B}\left(K_{i \Gamma}=0\right) \\
& +\beta^{2} \bar{G}_{\beta} \sum_{\substack{k_{1} \in \Lambda \backslash B \\
k_{1} \neq i, l}}\left|i-k_{1}\right|^{-2 \alpha d}\left\{I_{\left\{i, k_{1}\right\}, B}\left(C^{\prime}\right)+I_{\left\{l, k_{1}\right\}, B}\left(C^{\prime}\right)\right\},
\end{aligned}
$$

with $C^{\prime}$ standing for $\left\{K_{\{i, l\}, B}=0 ; K_{i m}=0, \forall m \leqq k_{1}\right\}$.

Next, we substitute the factor $I_{l, B}\left(K_{i \Gamma}=0\right)$ on the r.h.s. of $(2.70)$ by the r.h.s. of (2.ó6), with $C^{(n)}$ replaced by $C^{(n)} \cup\left\{K_{i \Gamma}=0\right\}$. This yields an upper bound for $I_{\{i, l\}, B}$ which is a sum of terms proportional to $I_{\left\{k, k^{\prime}\right\}, B}(\widetilde{C})$, with coefficients which are

$$
O\left(\beta^{2}|i-k|^{-2 \alpha d}\left|k-k^{\prime}\right|^{-2 \alpha d}\right) \text {. }
$$


By now the reader knows perfectly well how to extract a bound from such an inequality by iterating it indefinitely. The bound is convergent for $0 \leqq \beta<\beta_{0}$, with $\beta_{0}$ sufficiently small. Feeding this convergent bound for $I_{\left\{k, k^{\prime}\right\}, B}(C)$ back into inequality (2.66) finally yields a convergent bound for $I_{i, B}$. Both bounds have the form announced in Lemma 2.5(i), (ii), hence Lemma 2.5 is proven.

The analysis above clearly completes the proof of Proposition 2.4.

We now turn to the proof of a lower bound on $E\left(\mu\left(\sigma_{i} ; \sigma_{j}\right)^{2}\right)$. In a special case this has already been done in [11] by a somewhat elegant trick. Here we wish to show that a lower bound also emerges from our inductive construction. For simplicity, we only consider the special case, where there is no external magnetic field, so that $\mu_{\Lambda}\left(\sigma_{k}\right)=0$, for any $k$. We start from (2.50) which, in the present situation, takes the form

$$
I_{i, j}=I_{i, j}\left(K_{i j}=0\right)+\beta^{2}|i-j|^{-2 \alpha d} \cdot \int_{0}^{1} d s_{i j} \int_{0}^{s_{i j}} d s_{i j}^{\prime} E\left(\mathscr{F}_{i j}^{2}\left[\partial_{i j}^{2} \mu_{\Lambda}\left(\sigma_{i} \sigma_{j}\right)^{2}\right]_{s_{i j}}\right) .
$$

The point is now that

$$
\partial_{i j}^{2} \mu_{\Lambda}\left(\sigma_{i} \sigma_{j}\right)^{2}=2+O\left(\mu_{\Lambda}\left(\sigma_{i} \sigma_{j}\right)^{2}\right)
$$

By the upper bound

$$
E\left(\left.\mathscr{F}_{i j}^{2} \mu_{\Lambda}\left(\sigma_{i} \sigma_{j}\right)^{2}\right|_{S_{i j}^{\prime}}\right) \leqq \text { const } \cdot \beta^{2} .
$$

Hence, for $\beta$ small enough,

$$
\begin{aligned}
I_{i, j} & \geqq I_{i, j}\left(K_{i j}=0\right)+\beta^{2}|i-j|^{-2 \alpha d}\left\{E\left(\mathscr{F}_{i j}^{2}\right)-O\left(\beta^{2}\right)\right\} \\
& \geqq I_{i, j}\left(K_{i j}=0\right)+a^{\prime} \beta^{2}|i-j|^{-2 \alpha d},
\end{aligned}
$$

for some $a^{\prime}>0$.

Next, we use the upper bound on $\left|I_{i, j}\left(K_{i j}=0\right)\right|$ proven in (2.53)-(2.57), ... Since $K_{i j}=0$, the leading contribution to $\left|I_{i, j}\left(K_{i j}=0\right)\right|$ is of the form

$$
\text { const } \cdot \beta^{4} \sum_{k: i \neq k \neq j}|i-k|^{-2 \alpha d}|k-j|^{-2 \alpha d} \leqq b^{\prime} \beta^{4}|i-j|^{-2 \alpha d},
$$

and it is not hard to check that this is really an upper bound on $\left|I_{i, j}\left(K_{i j}=0\right)\right|$ if $\bar{\varrho} \equiv \varrho\left(\sigma_{k}\right)^{2}=0$. Hence

$$
I_{i, j} \geqq a^{\prime} \beta^{2}|i-j|^{-2 \alpha d}-b^{\prime} \beta^{4}|i-j|^{-2 \alpha d} \geqq a^{\prime \prime} \beta^{2}|i-j|^{-2 \alpha d},
$$

for some constant $a^{\prime \prime}$ which is strictly positive if $\beta$ is small enough.

The strategy for proving lower bounds on $I_{A, B}$ sketched here is quite general: One first extracts all leading terms contributing to $I_{A, B}$ explicitly. They have a decay like $d(A, B)^{-2 \alpha d}$, for $A$ and $B$ bounded. Then one uses the upper bounds established in the proof of Proposition 2.4 to estimate the remainder terms and to show they are of higher order in $\beta$ than the leading terms, but still decay like $d(A, B)^{-2 \alpha d}$. This will provide the desired lower bounds, provided $\beta$ is sufficiently small.

Well the strategy sounds simple. But it is actually quite cumbersome to implement it, except in the simple case treated above. The reader may wish to try out his skills on the next more complicated case. 


\section{High-Temperature Behaviour of $\mathrm{N}$-Vector Spin Glasses}

In this section we study $N$-vector spin glasses with Hamiltonian

$$
H\left(\sigma_{\Lambda}\right) \equiv H^{\mathscr{g}}\left(\sigma_{\Lambda}\right):=-\sum_{i, j \in \Lambda} J_{i j} \sigma_{i} \cdot \sigma_{j},
$$

where $\sigma_{i}$ is an $N$-component unit vector (i.e. $\sigma_{i} \in S_{N-1}$ ),

$$
\sigma_{i} \cdot \sigma_{j}=\sum_{m=1}^{N} \sigma_{i}^{m} \sigma_{j}^{m}, \quad J_{i j}=\mathscr{J}_{i j}|i-j|^{-\alpha d},
$$

with $\alpha>\frac{1}{2}$ and $\mathscr{J}_{i j}$ i.i.d. random variables. The distribution, $E$, of the variables $\mathscr{J}_{i j}$ has the properties

$$
E \mathscr{J}_{i j}=0, \quad\left|E \mathscr{J}_{i j}^{p}\right| \leqq \gamma^{p} p !, \quad p=2,3, \ldots,
$$

for some finite $\gamma$ (as in Sect. 2). For details concerning our notations, see Sect. 1.2. By $\mu_{\Lambda \varrho}$ we mean the finite-volume equilibrium state with boundary conditions $d \varrho\left(\sigma_{\Lambda^{\prime} \backslash \Lambda}\right)$, defined in (1.10). We also recall that

$$
\sigma_{A} \equiv \prod_{i \in \bar{A}} \prod_{m=1}^{N}\left(\sigma_{i}^{m}\right)^{n(m, i)}
$$

with $A=\{(i, m, n(m, i)): i \in \bar{A} \subset \Gamma, m=1, \ldots, N\}$; see (1.7).

Our main result in this section is

Proposition 3.1. Let $E$ be as in (3.3) and $\alpha>\frac{1}{2}$. Then there exists a positive constant $\beta_{0}$ such that, for $0 \leqq \beta<\beta_{0}$,

$$
\lim _{\substack{\Lambda \nearrow \Gamma \\ \Lambda^{\prime} \supset \Lambda}} E\left(\mu_{\Lambda \varrho} \sigma_{A}-\mu_{\Lambda \varrho^{\prime}} \sigma_{A}\right)^{2}=0
$$

and, for arbitrary, fixed $A$, the convergence is uniform in $\Lambda^{\prime} \supset \Lambda$ and in the choice of boundary conditions.

Let $\mu=\lim _{\Lambda \nearrow \Gamma} \mu_{\Lambda \varrho}$ be the limiting Gibbs state. Then

$$
E\left(\mu\left(\sigma_{A} ; \sigma_{B}\right)^{2}\right) \leqq \beta^{2} \bar{C}_{\beta} \xi_{\beta}^{|A|} \cdot|B| \cdot d(A, B)^{-2 \alpha d},
$$

for some finite constants $\bar{C}_{\beta}$ and $\xi_{\beta}$.

Remarks. As in the proof of Corollary 2.2, one shows that it follows from (3.5) that there exists a sequence of finite subsets, $\left\{\Lambda_{n}\right\}_{n=0}^{\infty}$, increasing to $\Gamma$ such that the limit

$$
\lim _{n \rightarrow \infty} \mu_{\Lambda_{n} \varrho} \equiv \mu
$$

exists and defines an extremal state which is independent of the b.c. $\varrho$. The second part of Proposition 3.1 states that $\mu$ is clustering.

Proof of Proposition 3.1. We start with the proof of (3.5). As in Sect. 2, we use the shorthand

$$
\left.I_{A}(C) \equiv E\left(\mu_{\Lambda \underline{\underline{\varrho}} \sigma_{A}}-\mu_{\Lambda \underline{\underline{\varrho^{\prime}}}} \sigma_{A}\right)^{2}\right|_{C}
$$

where $C$ is some condition imposed on the couplings $J_{i j}$. We set $I_{A}(\emptyset) \equiv I_{A}$. 
Our starting point is an identity analogous to (2.8):

$$
\begin{aligned}
I_{A}= & I_{A}\left(K_{k_{0} \Lambda^{c}}=0\right)+\sum_{j \in \Lambda^{\prime} \backslash \Lambda} \beta^{2}\left|k_{0}-j\right|^{-2 \alpha d} \\
& \times \int_{0}^{1} d s_{k_{0} j} \int_{0}^{s_{0_{0} j}} d s_{k_{0 j} j}^{\prime} E\left(\mathscr{J}_{k_{0} j}^{2}\left[\partial_{k_{0} j}^{2}\left(\mu_{\Lambda \varrho} \sigma_{A}-\mu_{A \underline{\varrho}^{\prime}} \sigma_{A}\right)^{2}\right]_{\left.C^{(0)}\right)}\right)^{2},
\end{aligned}
$$

where $k_{0}$ is the "least" site in $A$, in the order $\prec$, and $C^{(0)}$ is the condition $\left\{K_{k_{0} A^{c}}=0\right.$, $\left.K_{k_{0} i}=0, \forall i \prec j, S_{k_{0} j}^{\prime}\right\}$. See (1.14), (1.15), Sect. 1.2. (This is the analogue of Eqs. (8) and (9) in [11].) As in Sect. 2, (2.10), we conclude from (3.7) that

$$
I_{A} \leqq I_{A}\left(K_{k_{0} \Lambda^{c}}=0\right)+a \beta^{2} \sum_{j \in \Lambda^{c}}\left|k_{0}-j\right|^{-2 \alpha d},
$$

for some constant $a$ independent of $\Gamma, \varrho, \varrho^{\prime}, \alpha, \beta$, and $A$. The first term on the r.h.s. of (3.8) is expanded in a Taylor series in $\vec{J}_{k_{0} k_{1}}$ to second order, yielding

$$
\begin{aligned}
I_{A}\left(K_{k_{0} \Lambda^{c}}=0\right)= & \beta^{2} \sum_{\substack{k_{1} \in \Lambda \\
k_{1} \neq k_{0}}}\left|k_{0}-k_{1}\right|^{-2 \alpha d} \int_{0}^{1} d s_{k_{0} k_{1}} \int_{0}^{s_{k_{0} k_{1}}} d s_{k_{0} k_{1}}^{\prime} \\
& \times E\left(\left.\mathscr{J}_{k_{0} k_{1}}^{2} \partial_{k_{0} k_{1}}^{2}\left(\mu_{\Lambda \varrho} \sigma_{A}-\mu_{\Lambda \varrho^{\prime}} \sigma_{A}\right)^{2}\right|_{C^{(1)}}\right) \\
& +I_{A}\left(K_{k_{0} \Gamma}=0\right),
\end{aligned}
$$

where $C^{(1)}$ is given by $\left\{K_{k_{0} A^{c}}=0, K_{k_{0} i}=0, \forall i \prec k_{1}, S_{k_{0} k_{1}}^{\prime}\right\}$. It is useful to choose the order $\prec$ such that all sites in $\bar{A}$ are smaller than the sites in $\Lambda \backslash \bar{A}$.

We define

Then

$$
\bar{\varrho}\left(k_{0}, n\right)=\left(\int d \varrho(\sigma) \prod_{m=1}^{N}\left(\sigma^{m}\right)^{n\left(m, k_{0}\right)}\right)^{2} .
$$

$$
I_{A}\left(K_{k_{0} \Gamma}=0\right)=\bar{\varrho}\left(k_{0}, n\right) I_{A \backslash k_{0}}\left(K_{k_{0} \Gamma}=0\right) \leqq I_{A \backslash k_{0}}\left(K_{k_{0} \Gamma}=0\right) .
$$

If $A=\left\{k_{0}\right\}$ then $I_{A}\left(K_{k_{0} \Gamma}=0\right)=0$, and thus we set $I_{\emptyset}\left(K_{k_{0} \Gamma}=0\right)=0$. We conclude that we can get an upper bound on $I_{A}\left(K_{k_{0} A^{c}}=0\right)$ by induction in $|\bar{A}|$, the cardinality of $\bar{A}$, provided we can bound the first term on the r.h.s. of (3.9).

In Appendix A.1 we show that

$$
\left|\partial_{i k}^{2}\left(\mu_{\Lambda \underline{Q}} \sigma_{B}-\mu_{\Lambda \underline{Q}^{\prime}} \sigma_{B}\right)^{2}\right|_{C} \mid \leqq K U_{1}(B, i, k ; C),
$$

where

$$
\begin{aligned}
U_{1}(B, i, k ; C) \equiv & \left(\left[\mu_{\Lambda \varrho} \sigma_{B}\left(\sigma_{i} \cdot \sigma_{k}\right)^{2}-\mu_{\Lambda \varrho^{\prime}} \sigma_{B}\left(\sigma_{i} \cdot \sigma_{k}\right)^{2}\right]_{C}\right)^{2} \\
& +\left(\left[\mu_{\Lambda \varrho} \sigma_{B}\left(\sigma_{i} \cdot \sigma_{k}\right)-\mu_{\Lambda \varrho^{\prime}} \sigma_{B}\left(\sigma_{i} \cdot \sigma_{k}\right)\right]_{C}\right)^{2} \\
& +\left(\left[\mu_{\Lambda \varrho} \sigma_{B}-\mu_{\Lambda \varrho^{\prime}} \sigma_{B}\right]_{C}\right)^{2} \\
& +\left(\left[\mu_{\Lambda \varrho}\left(\sigma_{i} \cdot \sigma_{k}\right)^{2}-\mu_{\Lambda \varrho^{\prime}}\left(\sigma_{i} \cdot \sigma_{k}\right)^{2}\right]_{C}\right)^{2} \\
& +\left(\left[\mu_{\Lambda \varrho} \sigma_{i} \cdot \sigma_{k}-\mu_{\Lambda \varrho^{\prime}} \sigma_{i} \cdot \sigma_{k}\right]_{C}\right)^{2},
\end{aligned}
$$

and $K$ is some purely combinatorial constant. We use (3.12) and (3.13) to get an upper bound on the first term on the r.h.s. of (3.9). Subsequently, we iterate (3.9)-(3.13) for all terms appearing in $U_{1}\left(A, k_{0}, k_{1} ; C^{(1)}\right)$, but starting from the site 
$k_{1}$. The result is the inequality

$$
\begin{aligned}
I_{A} \leqq & a \beta^{2} \sum_{j \in \Lambda^{c}}\left|k_{0}-j\right|^{-2 \alpha d}+\sum_{j \in \Lambda^{c}} K \beta^{2} \sum_{\substack{k_{1} \in \Lambda \\
k_{1} \neq k_{0}}}\left|k_{0}-k_{1}\right|^{-2 \alpha d} \\
& \times \int_{0}^{1} d s_{k_{0} k_{1}} \int_{0}^{s_{k_{0} k_{1}}} d s_{k_{0} k_{1}}^{\prime} K \beta^{2}\left|k_{1}-j\right|^{-2 \alpha d} \\
& \times \int_{0}^{1} d s_{k_{1} j} \int_{0}^{s_{k_{1} j}} d s_{k_{1} j}^{\prime} \cdot E\left(\mathscr{J}_{k_{0} k_{1}}^{2} \mathscr{J}_{k_{1 j} j}^{2}\right) N\left(U_{1}\right)^{2} \cdot 2^{2} \\
& +K \beta^{2} \sum_{\substack{k_{1} \in \Lambda \\
k_{1} \neq k_{0}}}\left|k_{0}-k_{1}\right|^{-2 \alpha d} \int_{0}^{1} d s_{k_{0} k_{1}} \int_{0}^{s_{k_{0} k_{1}}^{\prime}} \cdot E\left(\mathscr{J}_{k_{0} k_{1}}^{2} U_{1}\left(A, k_{0}, k_{1} ; \widetilde{C}^{(1)}\right)\right. \\
& +I_{A \backslash k_{0}}\left(K_{k_{0} \Gamma}=0\right),
\end{aligned}
$$

where $\widetilde{C}^{(1)}$ is $C^{(1)} \vee\left\{K_{k_{1} \Lambda^{c}}=0\right\}$, and we have used (3.11) in the last term on the r.h.s. of (3.14). In the second term on the r.h.s. $N\left(U_{1}\right)=5$ is the number of terms appearing in $U_{1}$. The bound $N\left(U_{1}\right)^{2} \cdot 2^{2}$ comes from bounding $\left|\partial_{k, j}^{2} U_{1}\right|$ with the help of (3.12) and (3.13): Each term in $\partial_{k, j}^{2} U_{1}$ is estimated by five new terms, each of which is bounded by $2^{2}$.

We now continue our expansion by applying (3.9) to the terms in the factor $U_{1}\left(A, k_{0}, k_{1} ; \widetilde{C}^{(1)}\right)$, appearing in the third term on the r.h.s. of (3.14). This produces a term proportional to $U_{1}\left(A, k_{0}, k_{1} ; C^{(2)}\right)$, where $C^{(2)}$ is $\widetilde{C}^{(1)} \vee\left\{K_{k_{1} \Gamma}=0\right\}$. We now note that

$$
\begin{aligned}
& U_{1}\left(A, k_{0}, k_{1} ; C^{(2)}\right)=\left[\left(\mu \sigma_{A \backslash k_{1}}-\mu^{\prime} \sigma_{A \backslash k_{1}}\right)^{2}\left(\mu_{0} \prod_{m}\left(\sigma_{k_{1}}^{m}\right)^{n\left(m, k_{1}\right)}\right)^{2}\right. \\
& +\left\{\sum_{l, l^{\prime}}\left(\mu \sigma_{A \backslash k_{1}} \sigma_{k_{0}}^{l} \sigma_{k_{0}}^{l^{\prime}}-\mu^{\prime} \sigma_{A \backslash k_{1}} \sigma_{k_{0}}^{l} \sigma_{k_{0}}^{l^{\prime}}\right)\left(\mu_{0} \prod_{m}\left(\sigma_{k_{1}}^{m}\right)^{n\left(m, k_{1}\right)} \cdot \sigma_{k_{1}}^{l} \sigma_{k_{1}}^{l^{\prime}}\right)\right\}^{2} \\
& +\left\{\sum_{l}\left(\mu \sigma_{A \backslash k_{1}} \sigma_{k_{0}}^{l}-\mu^{\prime} \sigma_{A \backslash k_{1}} \sigma_{k_{0}}^{l}\right)\left(\mu_{0} \prod_{m}\left(\sigma_{k_{1}}^{m}\right)^{n\left(m, k_{1}\right)} \cdot \sigma_{k_{1}}^{l}\right)\right\}^{2} \\
& \left.+\left\{\sum_{l, l^{\prime}}\left(\mu \sigma_{k_{0}}^{l} \sigma_{k_{0}}^{l^{\prime}}-\mu^{\prime} \sigma_{k_{0}}^{l} \sigma_{k_{0}}^{l^{\prime}}\right)\left(\mu_{0} \sigma_{k_{1}}^{l} \sigma_{k_{1}}^{l^{\prime}}\right)\right\}^{2}+\left\{\sum_{l}\left(\mu \sigma_{k_{0}}^{l}-\mu^{\prime} \sigma_{k_{0}}^{l}\right)\left(\mu_{0} \sigma_{k_{1}}^{l}\right)\right\}^{2}\right]_{C^{(2)}}
\end{aligned}
$$

Here $\mu \equiv \mu_{\Lambda \underline{\underline{\varrho}}}, \mu^{\prime} \equiv \mu_{\Lambda \underline{\underline{\varrho}^{\prime}}}$ and

$$
d \mu_{0}(\sigma)=\prod_{j} d \varrho\left(\sigma_{j}\right) .
$$

If $d \varrho$ is the uniform measure on $S_{N-1}$ then

In this case we conclude, using

$$
\begin{gathered}
\mu_{0} \sigma_{k}^{l}=0, \\
\mu_{0} \sigma_{k}^{l} \sigma_{k}^{l^{\prime}}=\frac{1}{N} \delta_{l l^{\prime}} .
\end{gathered}
$$

$$
\sigma_{k}^{2}=\sum_{m} \sigma_{k}^{m} \sigma_{k}^{m}=1
$$

that the last two terms on the r.h.s. of (3.15) vanish. Moreover, if $k_{1} \notin A$,

$$
U_{1}\left(A, k_{0}, k_{1} ; C^{(2)}\right)=\left.K_{N}\left(\mu_{\Lambda \underline{\varrho}} \sigma_{A}-\mu_{\Lambda \underline{\varrho}^{\prime}} \sigma_{A}\right)^{2}\right|_{C^{(2)}},
$$


which also follows from (3.16). Here $k_{N}=1+N^{-2}$. These observations simplify our expansion for the models where $d \varrho$ is the uniform measure, but these simplifications are by no means essential. Returning to (3.14) and applying (3.9) to the third term on the r.h.s. of (3.14), we obtain the inequality

$$
\begin{aligned}
I_{A} \leqq & \left(a \beta^{2}+\left(5 K \beta^{2} E \mathscr{J}_{i j}^{2}\right)^{2} b\right) \sum_{j \in A^{c}}\left|k_{0}-j\right|^{-2 \alpha d} \\
& +\left(K \beta^{2}\right)^{2} \sum_{\substack{k_{1}, k_{2} \in A \\
k_{1} \neq k_{0}, k_{2} \neq k_{1}}}\left|k_{0}-k_{1}\right|^{-2 \alpha d}\left|k_{1}-k_{2}\right|^{-2 \alpha d} \\
& \times \int_{0}^{1} d s_{k_{0} k_{1}} \int_{0}^{s_{k_{0} k_{1}}} d s_{k_{0} k_{1}}^{\prime}\left\{\int_{0}^{s_{k_{0} k_{1}}} d s_{k_{1} k_{2}} \delta_{k_{2} k_{0}}+\int_{0}^{1} d s_{k_{1} k_{2}}\left(1-\delta_{k_{2} k_{0}}\right)\right\} \\
& \times \int_{0}^{s_{k_{1} k}} d s_{k_{1} k_{2}}^{\prime} E\left\{\mathscr{J}_{k_{0} k_{1}}^{2} \mathscr{J}_{k_{1} k_{2}}^{2} \partial_{k_{1} k_{2}}^{2} U_{1}\left(A, k_{0}, k_{1} ; \tilde{C}^{(2)}\right)\right\} \\
& +K \beta^{2} \sum_{k_{1} \in \Lambda}\left|k_{0}-k_{1}\right|^{-2 \alpha d} \int_{0}^{1} d s_{k_{0} k_{1}} \int_{0}^{s_{k_{0} k_{1}}} d s_{k_{0} k_{1}}^{\prime} \\
& \times E\left\{\mathscr{J}_{k_{0} k_{1}}^{2} U_{1}\left(A, k_{0}, k_{1} ; C^{(2)}\right)\right\}+I_{A \backslash k_{0}}\left(K_{k_{0} \Gamma}=0\right),
\end{aligned}
$$

where $\tilde{C}^{(2)}$ is $\left\{K_{k_{0} \Lambda^{c}}=0, K_{k_{0} i}=0, i \prec k_{1}, K_{k_{1} \Lambda^{c}}=0, K_{k_{1} i^{\prime}}=0, i^{\prime} \prec k_{2}, S_{k_{0} k_{1}}^{\prime}, S_{k_{1} k_{2}}^{\prime}\right\}$. We have used that

$$
\int_{0}^{1} d s_{k_{0} k_{1}} \int_{0}^{s_{k_{0} k_{1}}} d s_{k_{0} k_{1}}^{\prime} \int_{0}^{1} d s_{k_{1} j} \int_{0}^{s_{k_{1} j}} d s_{k_{1 j} j}^{\prime}=2^{-2},
$$

and that

$$
\sum_{k_{1} \neq k_{0}, j}\left|k_{0}-k_{1}\right|^{-2 \alpha d}\left|k_{1}-j\right|^{-2 \alpha d} \leqq b\left|k_{0}-j\right|^{-2 \alpha d} .
$$

Moreover, we have made the simplifying assumption that $E \mathscr{J}_{k l}^{2 n+1}=0$. Without this assumption a term $\propto E\left(\mathscr{J}_{k_{0} k_{1}}^{3}\right)$ would appear in the second term on the r.h.s. of (3.17) in case $k_{2}=k_{0}$. This is a minor complication which we do not propose to consider for a single reason: It would further complicate formulas which are already heavy.

If, in the second term on the r.h.s. of (3.17), $k_{2}$ coincides with $k_{0}$, we use that

$$
\int_{0}^{1} d s_{1} \ldots \int_{0}^{s_{n}-1} d s_{n} E\left(\mathscr{J}_{k l}^{n}\right) \leqq \gamma^{n}
$$

for $n=4$, in order to convince ourselves that the factor 4 ! in $E \mathscr{J}_{k_{0} k_{1}}^{4}$ is offset by the $s$ integrations.

Applying (3.12) and (3.13) to each term in $\partial_{k_{1} k_{2}}^{2} U_{1}$, we obtain the bound

$$
\left|\partial_{k_{1} k_{2}}^{2} U_{1}\left(A, k_{0}, k_{1} ; C\right)\right| \leqq K U_{2}\left(A, k_{0}, k_{1}, k_{2} ; C\right),
$$

where $U_{2}$ is a sum of $N\left(U_{2}\right)=5 N\left(U_{1}\right)=25$ terms, each of which has the form $\left.\left(\mu_{\Lambda \varrho} F-\mu_{\Lambda \varrho^{\prime}} F\right)^{2}\right|_{C}$ for a function, $F$, of the spins, $\sigma_{i}$, satisfying $\|F\|_{\infty} \leqq 1$, so that

$$
\left.\left(\mu_{\Lambda \varrho} F-\mu_{\Lambda \varrho^{\prime}} F\right)^{2}\right|_{C} \leqq 4
$$

After inserting (3.19) in the second term on the r.h.s. of (3.17), it becomes clear that our expansion can be iterated indefinitely by applying expansion steps, followed 
by an estimate of the form

$$
\left|\partial_{k_{n-1} k_{n}}^{2} U_{n-1}\left(A, k_{0}, \ldots, k_{n-1} ; C\right)\right| \leqq K U_{n}\left(A, k_{0}, \ldots, k_{n-1}, k_{n} ; C\right),
$$

with $N\left(U_{n}\right)=5 N\left(U_{n-1}\right)=$ number of terms of the form $\left.\left(\mu_{\Lambda \varrho} F-\mu_{\Lambda \varrho^{\prime}} F\right)^{2}\right|_{C}$ appearing in $U_{n}$, with $\|F\|_{\infty} \leqq 1$.

Thus, iterating (3.17), as described in (3.9), (3.14), and (3.19) -(3.21), we obtain the expansion

$$
\begin{aligned}
& I_{A} \leqq \beta^{2} C_{\beta} \sum_{j \in \Lambda^{c}}\left|k_{0}-j\right|^{-2 \alpha d}+I_{A \backslash k_{0}}\left(K_{k_{0} \Gamma}=0\right)
\end{aligned}
$$

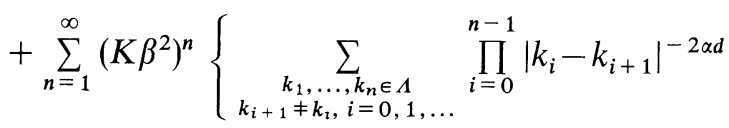

$$
\begin{aligned}
& \left.\times \int d_{n} \underline{S} E\left\{\left(\prod_{i=0}^{n-1} \mathscr{J}_{k_{i} k_{i+1}}^{2}\right) U_{n}\left(A, k_{0}, \ldots, k_{n} ; C^{(n)}\right)\right\}\right\},
\end{aligned}
$$

where the symbol $\int d_{n} \underline{s}(\cdot)$ stands for the integral

$$
\begin{aligned}
& \int_{0}^{1} d s_{k_{0} k_{1}} \int_{0}^{s_{k}} d s_{k_{0} k_{1}}^{\prime} \int_{0}^{s_{k_{0} k_{1}}} \int_{0}^{s_{k_{0} k_{1}}^{\prime}}\left(\int_{0}^{s_{k_{0} k_{1}}^{\prime}} d s_{k_{1} k_{2}} \delta_{k_{2} k_{0}}+\int_{0}^{1} d s_{k_{1} k_{2}}\left(1-\delta_{k_{2} k_{0}}\right)\right) \ldots \\
& \quad \ldots\left(\int_{0}^{s_{k_{n-2} k^{k} k_{n-1}}} d s_{k_{n-1} k_{n}} \delta_{k_{n} k_{n-2}}+\int_{0}^{1} d s_{k_{n-1} k_{n}}\left(1-\delta_{k_{n} k_{n-2}}\right)\right) \\
& \quad \times \int_{0}^{s_{k_{n-1} k_{n}}} d s_{k_{n-1} k_{n}}^{\prime}(\cdot)
\end{aligned}
$$

the $U_{n}$ 's are defined inductively in (3.21), and the conditions $C^{(n)}$ are given by

$$
\left\{K_{k_{m} \Lambda^{c}}=0 ; K_{k_{m} i}=0, \forall i \prec k_{m+1}\right\}_{m=0}^{n-1} \vee\left\{K_{k_{n} \Gamma}=0\right\} \text {. }
$$

Finally, the constant $C_{\beta}$ is given by

$$
C_{\beta}=a \beta^{2}+\left(5 K \beta^{2} \gamma^{2}\right)^{2} b\left(\sum_{n=0}^{\infty}\left(5 K \beta^{2} \gamma^{2} b\right)^{n}\right) .
$$

It is easy to prove absolute convergence of the expansion in (3.22): Every

$$
U_{n}\left(A, k_{0}, \ldots, k_{n} ; C^{(n)}\right)
$$

is given by $5^{n}$ terms, each of the form $\left.\left(\mu_{\Lambda \varrho} F-\mu_{\Lambda \varrho^{\prime}} F\right)^{2}\right|_{C^{(n)}}$, with $\|F\|_{\infty} \leqq 1$. Hence

$$
\left|U_{n}\left(A, k_{0}, \ldots, k_{n} ; C^{(n)}\right)\right| \leqq 5^{n} \cdot 4 \text {. }
$$

Hence

$$
E\left\{\left(\prod_{i=0}^{n-1} \mathscr{J}_{k_{i} k_{k_{i}+1}}^{2}\right) U_{n}\left(A, k_{0}, \ldots, k_{n} ; C^{(n)}\right)\right\} \leqq 5^{n} \cdot 4 E\left(\prod_{i=0}^{n-1} \mathscr{J}_{k_{\imath} k_{l+1}}^{2}\right)
$$

This bound is $\underline{s}$-independent. The integral $\int d_{n} \underline{s} 1$ has the value

$$
\int d_{n} \underline{\underline{s}} 1=\prod_{(i j)}\left[\left(2 n_{i j}\right) !\right]^{-1},
$$


where $n_{i j}$ is the number of times we have expanded in the couplings $J_{i j}$ (to second order). Furthermore,

$$
E\left(\prod_{i=0}^{n-1} \mathscr{F}_{k_{i} k_{i+1}}^{2}\right) \leqq \gamma^{2 n} \prod_{(i j)}\left(2 n_{i j}\right) !
$$

See also (3.18). Finally, we use that

$$
\sum_{k_{i} \neq k_{i-1}, k_{i+1}}\left|k_{i-1}-k_{i}\right|^{-2 \alpha d}\left|k_{i}-k_{i+1}\right|^{-2 \alpha d} \leqq b\left(\left|k_{i-1}-k_{i+1}\right|+1\right)^{-2 \alpha d} .
$$

From (3.26) - (3.30) we conclude that the infinite series on the r.h.s. of (3.22) is dominated by the geometric series

$$
\sum_{n=1}^{\infty} \sum_{k_{n} \in A}\left(K \beta^{2}\right)^{n} \gamma^{2 n} 5^{n} \cdot 4 b^{n-1}\left(\left|k_{0}-k_{n}\right|+1\right)^{-2 \alpha d},
$$

which is absolutely convergent for $0 \leqq \beta<\beta_{0}$, with $\beta_{0}$ small enough. It is, therefore, a safe starting point for further expansions which we describe next:

The $n$-th term on the r.h.s. of (3.22) is expanded in the couplings $J_{k_{n-1} i}, i \in \Lambda$. [By (3.24), the couplings $J_{k_{n-1} j}, j \in \Lambda^{c}$, have already been turned off.] The term of order 0 in $J_{k_{n-1} i}$ is proportional to

$$
U_{n}\left(A, k_{0}, \ldots, k_{n-1}, k_{n} ; C^{(n)} \vee\left\{K_{k_{n-1} \Gamma}=0\right\}\right) .
$$

The second order term in $J_{k_{n-1} k_{1}^{1}}, k_{1}^{1} \in \Lambda$, is proportional to

$$
\partial_{k_{n-1} k_{1}^{1}}^{2} U_{n}\left(A, k_{0}, \ldots, k_{n-1}, k_{n}, C^{(n)} \vee \ldots\right)
$$

which is bounded, as in (3.21), by a term

$$
K U_{n, 1}\left(A, k_{0}, \ldots, k_{n-1}, k_{n}, k_{1}^{1}, C^{(n)} \vee \ldots\right) .
$$

It bears an additional small factor $\propto \beta^{2}\left|k_{n-1}-k_{1}^{1}\right|^{-2 \alpha d}$. Now we expand in the couplings $\mathscr{J}_{k_{1}^{1}}, j \in \Lambda^{c}$. The two expansion steps yield the new convergence factor

$$
\sum_{j \in \Lambda^{c}} \sum_{\substack{k \in \Lambda \\ k 1 \neq k_{n-1}}} a \beta^{2}\left(K \beta^{2}\right)\left|k_{n-1}-k_{1}^{1}\right|^{-2 \alpha d}\left|k_{1}^{1}-j\right|^{-2 \alpha d},
$$

which tends to 0 , as $\Lambda \nearrow \Gamma$.

The next step is to expand the term

$$
K U_{n, 1}\left(A, k_{0}, \ldots, k_{n}, k_{1}^{1}, C^{(n)} \vee\left\{K_{k_{1}^{1} \Lambda^{c}}=0\right\}\right)
$$

to second order in $J_{k_{1}^{1} k_{2}^{1}}, k_{2}^{1} \in \Lambda$, and so on. This process can be continued in a way very similar to how we continued (3.14) to obtain (3.22). After having completed our expansion in $J_{k_{n-1} k_{1}^{1}}, J_{k_{1}^{1} k_{2}}^{1}, \ldots$ we continue our construction by expanding in $J_{k_{n-2} k_{1+1}^{1}}, J_{k_{l+1}^{1} k_{l+2}^{1}}, \ldots$, always to second order and using (3.21) to estimate the second order remainder in the Taylor expansion. Eventually, we shall continue our expansion at some site $k_{m}^{1}$, expanding in $J_{k_{m}^{1} k_{1}^{2}}, k_{1}^{2} \in \Lambda$ to second order. By induction we can easily establish the structure of the general term emerging from the construction described above: Every such term is labelled by a general, abstract tree, $T$, rooted in a vertex, $O$, with branches ordered in generations. Given a tree, $T$, let $\mathscr{V}(T)$ denote the set of all vertices in $T$ and $\mathscr{L}(T)$ the set of all lines in $T$. Moreover, by $\tau$ we denote an arbitrary map from $\mathscr{V}(T)$ into $\Gamma$ such that $\tau(0)=k_{0}$, 
and $\tau(k) \neq \tau(l)$ if $(k l) \in \mathscr{L}(T)$. There are two types of such maps: Maps, $\tau$, which map one vertex $l \in \mathscr{V}(T)$ to a site $j=\tau(l) \in \Lambda^{c}$, called maps of type 1, and maps which map all of $\mathscr{V}(T)$ into $\Lambda$, called of type 2 .

A term in the expansion labelled by a map of type 1 has the form

$$
\begin{aligned}
& \left(K \beta^{2}\right)^{\left|\mathscr{L}\left(T^{\prime}\right)\right|} \sum_{\substack{j \in \Lambda^{c} \\
\tau^{-1}(j) \in \mathscr{V}(T)}} \sum_{\substack{k \in \Lambda: \\
k=\tau(i), i \in \mathscr{V}(T)}} \prod_{(i l) \in \mathscr{L}(T)}|\tau(i)-\tau(l)|^{-2 \alpha d} \\
& \times \int d_{T \underline{S}} E\left\{\left(\prod_{(i l) \in \mathscr{L}(T)} \mathscr{J}_{\tau(i) \tau(l)}^{2}\right) \partial_{j^{\prime} j}^{2} U\left(A, T^{\prime}, \tau ; C\left(T^{\prime}, \tau\right)\right)\right\}
\end{aligned}
$$

where $\left(j^{\prime} j\right)=(\tau(m) \tau(n)),(m n) \in \mathscr{L}(T)$, with $\tau(m) \in \Lambda, \tau(n) \in \Lambda^{c}$, and $T^{\prime}$ is the tree obtained from $T$ by deleting the line $(m n)$.

A term labelled by a map of type 2 has the form

$$
\begin{aligned}
& \left(K \beta^{2}\right)^{|\mathscr{L}(T)|} \sum_{\substack{k \in \Lambda: \\
k=\Lambda(i), i \in \mathscr{V}(T)}} \prod_{(i l) \in \mathscr{L}(T)}|\tau(i)-\tau(l)|^{-2 \alpha d} \\
& \times \int d_{T} E\left\{\left(\prod_{(i l) \in \mathscr{L}(T)} \mathscr{J}_{\tau(i) \tau(l)}^{2}\right) U(A, T, \tau ; C(T, \tau))\right\},
\end{aligned}
$$

where $C(T, \tau)$ contains the conditions

$$
\left\{K_{k \Gamma}=0, \forall k=\tau(i), i \in \mathscr{V}(T)\right\} .
$$

The integrations $\int d_{T} s(\cdot)$ extend over domains which can be read off from (3.23): If the pair of sites $(k l)$ is covered by the map $\tau$ by $n$ different lines in $\mathscr{L}(T)$ the integration is given by

$$
\int_{0}^{1} d s_{k l} \int_{0}^{s_{k l}} d s_{k l}^{1} \ldots \int_{0}^{s_{k l}^{2 n-1}} d s_{k l}^{2 n}(\cdot)
$$

These integrations will yield a convergence factor $[(2 n) !]^{-1}$ which offsets the divergence factor in

$$
E \mathscr{J}_{k l}^{2 n} \leqq \gamma^{2 n}(2 n) ! .
$$

Our expansion has achieved the following goals:

(1) It contains terms displaying interactions between the site $k_{0} \in \bar{A}$ and sites $j \in \Lambda^{c}$ (type 1). The sum of these terms will turn out to tend to 0 like $\sum_{j \in \Lambda^{c}}\left|k_{0}-j\right|^{-2 \alpha d}$
as $\Lambda \nearrow \Gamma$.

(2) It contains remainder terms (type 2) with the properties that $\tau(\mathscr{V}(T)) \subseteq \Lambda$ and that all interactions between any site $k$ in $\tau(\mathscr{V}(T))$ and all other sites $i \in \Gamma$ are turned off. Thus, these remainder terms do not contain any interaction, $J_{k l}$, anymore, with $k \in \tau(\mathscr{V}(T))$ and $l \notin \tau(\mathscr{V}(T))$. Put differently, only interactions $J_{k l}$ with $(k l) \notin \tau(\mathscr{L}(T))$ have not been removed from the expectations, yet. Hence the remainder terms can be written as sums of terms which factorize into a product of two factors one of which is proportional to $\mu_{0}\left(\prod_{m=1}^{N}\left(\sigma_{k_{0}}^{m}\right)^{n(m)}\right)$, for some nonnegative integers $n(m)$, and the second factor is an expectation of $\prod_{i \in \bar{A} \backslash \bar{A}_{0}}\left(\prod_{m=1}^{N}\left(\sigma_{i}^{m}\right)^{n(m, i)}\right)$ in a state not containing any interactions between $\bar{A}_{0}$ and its complement, anymore, and $\bar{A}_{0} \supseteqq\left\{k_{0}\right\}$. We then estimate $\left|\mu_{0}\left(\prod_{m=1}^{N}\left(\sigma_{k_{0}}^{m}\right)^{n(m)}\right)\right|$ from above by 1 . In the second factor we shall pick a site $k_{0}^{\prime} \in \bar{A} \backslash \bar{A}_{0}\left(k_{0}^{\prime} \neq k_{0}\right)$, and repeat the entire expansion described above. This will actually permit us to induct in the cardinality of $|\bar{A}|$, just as in the Ising spin glass. 
All that remains to be done is to prove convergence of our scheme and an upper bound on terms of type 1 which tends to 0 , as $\Lambda \nearrow \Gamma$.

As already mentioned, the branches of all trees labelling terms in our expansion come in generations: The first generation is a walk $\left(k_{0} k_{1}\right)\left(k_{1} k_{2}\right) \ldots\left(k_{n-1} k_{n}\right)$. The second generation consists of branches growing out of $k_{n-1}, k_{n-2}, \ldots, k_{1}, k_{0}$. The third generation consists of branches growing out of sites which belong to branches of the second generation, but are not endpoints; and so on. In order to prove convergence, we shall successively "roll up the branches" of the trees labelling the terms in our expansion, starting always with the branches of the largest generation not rolled up, yet, and going back to branches of lower generations, one by one. "Rolling up a branch of generation $i+1$ " means that we first sum over its endpoint $k_{n_{2}}^{i}$, without respecting any constraints, like $k_{n_{2}}^{i}$ $=k_{l}^{i-m}, 1 \leqq m \leqq i$, then we sum over $k_{n_{2}-1}^{i}$ without respecting constraints, finally over $k_{n_{1}+1}^{i}$, with $n_{1}<n_{2}$. The site $k_{n_{1}}^{i}$ is the root of this branch and is a site of a branch in the $i^{\text {th }}$ generation. It is not yet summed over. In rolling up branches we make use of the following inequalities:

$$
|i-j|^{-2 \alpha d} \leqq \delta(|i-j|+1)^{-2 \alpha d}
$$

for some finite $\delta$, provided $i \neq j$.

$$
\sum_{k \in A}(|i-k|+1)^{-2 \alpha d} \leqq b^{\prime},
$$

for some finite constant $b^{\prime}$, and, in rolling up branches in terms of type 1 with an endpoint $j \in \Lambda^{c}$, we also use

$$
\sum_{k}(|l-k|+1)^{-2 \alpha d}(|k-i|+1)^{-2 \alpha d} \leqq b^{\prime \prime}(|l-i|+1)^{-2 \alpha d} .
$$

We recall that $\alpha>\frac{1}{2}$ which is used in (3.43) and (3.44). We set $b \equiv \delta \cdot \max \left(b^{\prime}, b^{\prime \prime}\right)$.

These estimates, combined with (3.21), (3.28) [or (3.40)], and (3.29) [or (3.41)], show that the weight of a line $(i l) \in \mathscr{L}(T)$ can be estimated by

$$
5 K \beta^{2} \gamma^{2} b
$$

with $K$ some finite combinatorial constant (depending on $N$ ). Rolling up one generation of branches growing out of the site $i$ yields an additional factor of

$$
(1-c(\beta))^{-1}
$$

where $c(\beta)$ is given by (3.45), so the effective weight of line $(i l)$, after rolling up a branch emanating from $i$ in the next higher generation, is given by

$$
c(\beta)(1-c(\beta))^{-1} .
$$

Now (il) belongs to some branch which may be rolled up next, and so on. We conclude that, in order to calculate the effective weight of lines (il) in the lowest (first) generation, we must solve the recursion relation

$$
c_{n+1}=c(\beta)\left(1-c_{n}\right)^{-1}, \quad n=0,1,2, \ldots,
$$

where $c_{0}=c(\beta)=5 K \beta^{2} \gamma^{2} b$. Here $c_{n}$ is the effective weight of a line after rolling up $n$ higher generations. Clearly, (3.48) has a solution $c^{*}=\operatorname{const} \beta^{2}<\infty$, provided $c(\beta)$ is small enough, i.e. for $0 \leqq \beta<\beta_{0}$ with $\beta_{0}$ small enough. 
These considerations establish the convergence of our scheme. It is now easy to complete the proof of the first part of Proposition 3.1, i.e., by induction in $|\bar{A}|$, we conclude that

$$
\lim _{\substack{\Lambda \nearrow \Gamma \\ \Lambda^{\prime} \supset \Lambda}} E\left(\mu_{\Lambda \underline{\varrho}} \sigma_{A}-\mu_{\Lambda \underline{\varrho}^{\prime}} \sigma_{A}\right)^{2}=0,
$$

for arbitrary $A$. This is what is claimed in (3.5). We finally turn to a sketch of our Proof of Clustering. Our proof of clustering for $N$-vector spin glasses is very similar to that for Ising spin glasses. We just substitute the Ising expansion by the general expansion developed above. Let $\bar{A}$ be a finite subset of $\Gamma$, and let $\bar{B}$ be a subset of $\Gamma$ with $|\bar{B}| \geqq|\bar{A}|$. We choose a site $k_{0} \in \bar{A}$ and write

$$
\begin{aligned}
E\left(\mu_{\Lambda \underline{\varrho}}\left(\sigma_{A} ; \sigma_{B}\right)^{2}\right)= & \left.E\left(\mu_{\Lambda \underline{\varrho}}\left(\sigma_{A} ; \sigma_{B}\right)^{2}\right)\right|_{K_{k_{0} B}=0} \\
& +\beta^{2} \sum_{j \in B}\left|k_{0}-j\right|^{-2 \alpha d} \int_{0}^{1} d s_{k_{0} j} \int_{0}^{s_{k_{0} j}} d s_{k_{0} j}^{\prime} \\
& \times E\left\{\mathscr{J}_{k_{0} j}^{2} \partial_{k_{0} j}^{2}\left[\mu_{\Lambda \varrho}\left(\sigma_{A} ; \sigma_{B}\right)^{2}\right]_{K_{K_{k_{0} j}}}=0, i \prec j\right\} .
\end{aligned}
$$

The second term on the r.h.s. of (3.49) is bounded by $a \beta^{2} \sum_{j \in B}\left|k_{0}-j\right|^{-2 \alpha d}$ which has the desired decay. So, it suffices to consider the first term. We perform the expansion step

$$
\begin{aligned}
\left.E\left(\mu_{\Lambda \underline{\varrho}}\left(\sigma_{A} ; \sigma_{B}\right)^{2}\right)\right|_{K_{k_{0} B}=0}= & \left.E\left(\mu_{\Lambda \varrho}\left(\sigma_{A} ; \sigma_{B}\right)^{2}\right)\right|_{K_{k_{0} \Gamma}=0} \\
& +\beta^{2} \sum_{\substack{k_{1} \in B^{c} \\
k 1 \neq k_{0}}}\left|k_{0}-k_{1}\right|^{-2 \alpha d} \int_{0}^{1} d s_{k_{0} k_{1}} \int_{0}^{s_{k_{0} k_{1}}} d s_{k_{0} k_{1}}^{\prime} \\
& \times E\left\{\mathscr{J}_{k_{0} k_{1}}^{2} \partial_{k_{0} k_{1}}^{2}\left[\mu_{\Lambda \varrho}\left(\sigma_{A} ; \sigma_{B}\right)^{2}\right] C\right\}
\end{aligned}
$$

where $C$ is the condition $\left\{K_{k_{0} B}=0, K_{k_{0} i}=0\right.$, for $\left.i \prec k_{1}, S_{k_{0} k_{1}}^{\prime}\right\}$. Terms corresponding to sites $k_{1} \in \Lambda^{c}$ are treated as in (3.7), (3.8). If $k_{1} \in \Lambda$ we use inequality (A.6) in Appendix A.2, i.e.

$$
\left|\partial_{k_{0} k_{1}}^{2} \mu_{\Lambda \varrho}\left(\sigma_{A} ; \sigma_{B}\right)^{2}\right|_{C} \mid \leqq K U_{1}\left(A, B, k_{0}, k_{1} ; C\right),
$$

where $K$ is a finite, combinatorial constant and $U_{1}$ is defined in (A.7). It is a sum of five terms of the form $\mu_{\Lambda \varrho}(F ; G)^{2}$, where $F$ and $G$ are polynomials in $\left\{\sigma_{i}^{m}\right\}$ (with coefficients $=1$ ). Using that

we obtain

$$
\left.E\left(\mu_{\Lambda \underline{\varrho}}\left(\sigma_{A} ; \sigma_{B}\right)^{2}\right)\right|_{K_{k_{0} \Gamma}=0} \leqq\left. E\left(\mu_{\Lambda \underline{\varrho}}\left(\sigma_{A \backslash k_{0}} ; \sigma_{B}\right)^{2}\right)\right|_{K_{k_{0} \Gamma}=0},
$$

$$
\begin{aligned}
E\left(\mu_{\underline{\varrho}}\left(\sigma_{A} ; \sigma_{B}\right)^{2}\right)= & a \beta^{2} \sum_{j \in B}\left|k_{0}-j\right|^{-2 \alpha d} \\
& +a^{\prime} \beta^{2} \sum_{j \in \Lambda^{c}}\left|k_{0}-j\right|^{-2 \alpha d}+\left.E \mu_{\Lambda \underline{\underline{g}}}\left(\sigma_{A \backslash k_{0}} ; \sigma_{B}\right)^{2}\right|_{K_{k_{0}}=0} \\
& +K \beta^{2} \sum_{\substack{k_{1} \in \Lambda \backslash B \\
k_{1} \neq k_{0}}}\left|k_{0}-k_{1}\right|^{-2 \alpha d} \int_{0}^{1} d s_{k_{0} k_{1}} \int_{0}^{s_{k_{0} k_{1}}} d s_{k_{0} k_{1}}^{\prime} \\
& \times E \mathscr{J}_{k_{0} k_{1}}^{2} U_{1}\left(A, B, k_{0}, k_{1} ; C\right),
\end{aligned}
$$


where $C$ is the condition defined after (3.50). By removing the couplings between $k_{1}$ and $\Lambda^{c}$ we obtain an inequality very similar to (3.14). We may continue our expansion steps as in (3.17)-(3.22), using an analogue of (3.21). This permits us to use induction in the volume $|\bar{A}|$ of $\bar{A}$. The details of our scheme to prove clustering are almost identical to our constructions in (3.17)-(3.41), and it is not worth to describe them once more. This completes the proof of Proposition 3.1.

In the special case of $E\left(\mu_{\Lambda}\left(\sigma_{i} ; \sigma_{j}\right)^{2}\right)$ (as well as other special, truncated correlations), our expansion also provides a lower bound of the form

$$
E\left(\mu_{\Lambda}\left(\sigma_{i} ; \sigma_{j}\right)^{2}\right) \geqq \tilde{a} \beta^{2}|i-j|^{-2 \alpha d} .
$$

This can be shown by arguments very similar to those in (2.71)-(2.76).

We wish to note that, by carefully tracing constants in the expansions of this section, we can show that, in zero magnetic field and with $d \varrho(\sigma)$ given by the uniform measure on $S_{N-1}$, our expansions converge for

as expected.

$$
0 \leqq \beta \leqq \operatorname{const} N,
$$

\section{Extensions of Results, Open Problems}

Our methods can be extended in several directions.

(1) Asymptoticity of the high-temperature expansion: The results proven in this paper clearly show that expectations

$$
E \mu_{\Lambda \underline{Q}}\left(\sigma_{A}\right)^{2}
$$

are continuously differentiable in $\beta$, for $\beta \in\left(-\beta_{0}, \beta_{0}\right)$. The first derivative vanishes at $\beta=0$. It is not hard to check that, if $J_{i j}$ satisfies (1.3), (1.4), with $\alpha>\frac{1}{2}$, then all terms in a Taylor expansion of $E \mu_{A \underline{Q}}\left(\sigma_{A}\right)^{2}$ in powers of $\beta$ are finite. In order to prove that the high-temperature expansion, though presumably divergent, is asymptotic, it would suffice to prove an upper bound $\sim O\left(\beta^{n+\varepsilon}\right), \varepsilon>0$, on the remainder term in the $n$-th order Taylor expansion of $E \mu_{A g}\left(\sigma_{A}\right)^{2}$ in powers of $\beta$. Although we have not checked the details, we expect that this can be done with the help of our expansion methods. For this purpose we have to extend our expansion methods to expressions of the form

$$
E \mu_{\Lambda \underline{\underline{Q}}}\left(\sigma_{A_{1}} ; \ldots ; \sigma_{A_{k}}\right) \mu_{\Lambda \underline{\underline{Q}}}\left(\sigma_{A_{k+1}} ; \ldots ; \sigma_{A_{n}}\right) .
$$

We do not see any obstacles against doing just that, although a detailed study of such expressions would admittedly be rather complicated.

In a proof of asymptoticity of the high temperature expansion, and other applications of our methods, it may be technically convenient to approximate a spin glass with long-range exchange couplings by one with short-range couplings, e.g. by multiplying $J_{i j}$ by

$$
g_{\varepsilon}(i, j) \equiv g(\varepsilon|i-j|),
$$

where $g$ is a $C^{\infty}$ function of compact support, and $\varepsilon>0$ will be chosen to be small. That the correlations of the approximate model converge to the correlations of the original model is the content of the next remark. 
(2) Approximation by short-range interactions: Let $g_{\varepsilon}$ be as defined in (4.2), and let $J_{i j}$ be as in (1.3), (1.4). Consider an $N$-vector spin glass, $N \geqq 1$. Let $\mu \equiv \mu_{\Lambda \underline{\rho}}^{\beta \Phi}$ be given by (1.10), and $\mu^{\varepsilon} \equiv \mu_{\Lambda \underline{\varrho}}^{\beta g_{\mathcal{E}} \mathscr{I}}$. Then

$$
\lim _{\varepsilon \searrow 0} E\left(\mu \sigma_{A}-\mu^{\varepsilon} \sigma_{A}\right)^{2}=0
$$

To prove (4.3), we use an expansion similar to that in Sect. 3. Its purpose is to successively turn off interactions between a site $k_{0} \in \bar{A}$ and the sites in $\Lambda \backslash\left\{k_{0}\right\}$. This will permit us to use induction in $|\bar{A}|$. On the basis of the techniques developed in Sect. 3 , it is not hard to show that the leading term is bounded by

$$
O\left(\beta^{2} \sum_{i \neq j \in \Gamma}|i-j|^{-2 \alpha d}\left(1-g_{\varepsilon}(i, j)\right)\right)
$$

and that the remaining terms sum up to a quantity which is even smaller than (4.4). Hence (4.3) follows. [In a proof of asymptoticity of the high-temperature expansion to $O\left(\beta^{n}\right)$, one would work with an $\varepsilon$ so small that $(4.4)$ is $O\left(\beta^{n+1}\right)$.]

(3) Variants of cluster- and phase-space-cell expansions: Of course, the expansion methods developed in this paper can be applied to nonrandom systems, as well, e.g. to Euclidean field theory. In a deterministic system, our expansion steps will consist of first-order Taylor expansions in the couplings between distant parts of the system. If we expand normalized quantities (correlations) we use superstability estimates [15] to bound and simplify the result of an expansion step. But we may also use our methods to systematically expand unnormalized quantities (correlations multiplied by a partition function) and then use estimates on ratios of partition functions, like those in [16], or exponentiate expansions of unnormalized quantities, using the algebraic formalism [17]. In this form, our expansion methods are compatible with complex coupling constants, and we can extend convergence proofs into the complex plane of some coupling parameters. These ideas would permit us to analyze, for example, the Euclidean Green function in a class of superrenormalizable field theory models and prove clustering. But such results are not new. More attractive extensions would be to combine our methods with an approach to cluster expansions, due, originally, to Glimm and Spencer, described by Brydges in [18], or with a form of phase-space-cell expansions proposed by Battle and Federbush [19]. The virtue of our methods is to avoid "over-expanding," i.e. to replace expansions producing lots of terms by expansions generating fewer terms, all of which can be labelled by abstract trees and can be estimated quite easily by "rolling up branches" (see Sect. 3).

(4) Renormalization group transformations: A single renormalization group transformation in a lattice or Euclidean field theory poses problems somewhat reminiscent of those met in the analysis of disordered spin systems (the block field variables play the role of random couplings). This was already observed in [10]. We think that the methods developed in Sect. 3, in a form like that sketched in remark (3) above, combined with the ideas in [10], form a very general machine to perform renormalization group transformations. Since we do not illustrate the workings of this machine in some examples, we must, of course, leave to the reader the benefit of the doubt. 


\section{Appendix A.1. Proof of Inequalities (2.13) and (3.12)}

Let $B \subset \Gamma \times\{1, \ldots, N\} \times \mathbb{N}$. The projection of $B$ onto $\Gamma$ is denoted by $\bar{B}$ and is assumed to be a bounded set. Let $\sigma_{B}$ be as in (1.7). Let $\mu \equiv \mu_{\Lambda \underline{Q}}$ and $\mu^{\prime} \equiv \mu_{\Lambda \underline{\varrho}^{\prime}}$. By direct calculation we can see that

$$
\begin{aligned}
\left.\partial_{i k}^{2}\left(\mu \sigma_{B}-\mu^{\prime} \sigma_{B}\right)^{2}\right|_{C}= & \partial_{i k}\left[2\left(\mu \sigma_{B}-\mu^{\prime} \sigma_{B}\right) \cdot\left\{\mu\left(\sigma_{B} ; \sigma_{i} \sigma_{k}\right)-\mu^{\prime}\left(\sigma_{B} ; \sigma_{i} \sigma_{k}\right)\right\}\right]_{C} \\
= & 2\left[\mu\left(\sigma_{B} ; \sigma_{i} \sigma_{k}\right)-\mu^{\prime}\left(\sigma_{B} ; \sigma_{i} \sigma_{k}\right)\right]_{C}^{2} \\
& +\left.2\left(\mu \sigma_{B}-\mu^{\prime} \sigma_{B}\right)\right|_{C} \partial_{i k}\left[\mu\left(\sigma_{B} ; \sigma_{i} \sigma_{k}\right)-\mu^{\prime}\left(\sigma_{B} ; \sigma_{i} \sigma_{k}\right)\right]_{C} .
\end{aligned}
$$

The first term on the r.h.s. of (A.1) has the following upper bound:

$$
\begin{aligned}
& 2\left[\mu\left(\sigma_{B} ; \sigma_{i} \sigma_{k}\right)-\mu^{\prime}\left(\sigma_{B} ; \sigma_{i} \sigma_{k}\right)\right]_{C}^{2} \\
& \quad=2\left[\left(\mu \sigma_{B} \sigma_{i} \sigma_{k}-\mu^{\prime} \sigma_{B} \sigma_{i} \sigma_{k}\right)-\left(\mu \sigma_{B}-\mu^{\prime} \sigma_{B}\right) \mu \sigma_{i} \sigma_{k}-\left(\mu \sigma_{i} \sigma_{k}-\mu^{\prime} \sigma_{i} \sigma_{k}\right) \mu^{\prime} \sigma_{B}\right]_{C}^{2} \\
& \quad \leq 2 \cdot 3\left[\left(\mu \sigma_{B}-\mu^{\prime} \sigma_{B}\right)^{2}+\left(\mu \sigma_{B} \sigma_{i} \sigma_{k}-\mu^{\prime} \sigma_{B} \sigma_{i} \sigma_{k}\right)^{2}-\left(\mu \sigma_{i} \sigma_{k}-\mu^{\prime} \sigma_{i} \sigma_{k}\right)^{2}\right]_{C}, \quad(A)
\end{aligned}
$$

where we have used the trivial inequality $(a+b+c)^{2} \leqq 3\left(a^{2}+b^{2}+c^{2}\right)$ and the fact that

$\left|\left[\mu \sigma_{i} \sigma_{k}\right]_{C}\right| \leqq 1$ and $\left|\left[\mu^{\prime} \sigma_{B}\right]_{C}\right| \leqq 1$

Remark. If $|B|$ is odd and $d \varrho(\sigma)$ is even in $\sigma$, then $\mu_{A} \sigma_{B}=0$ (for 0-b.c.), and the last term on the r.h.s. of (A.2) is absent.

To bound the last term on the r.h.s. of (A.1), we note that

$$
\begin{aligned}
\partial_{i k}[ & \left.\mu\left(\sigma_{B} ; \sigma_{i} \sigma_{k}\right)-\mu^{\prime}\left(\sigma_{B} ; \sigma_{i} \sigma_{k}\right)\right]_{C} \\
= & {\left[\mu \sigma_{B}\left(\sigma_{i} \sigma_{k}\right)^{2}-\mu \sigma_{B} \sigma_{i} \sigma_{k} \mu \sigma_{i} \sigma_{k}\right.} \\
& \left.-\mu\left(\sigma_{B} ; \sigma_{i} \sigma_{k}\right) \mu \sigma_{i} \sigma_{k}-\mu \sigma_{B} \mu\left(\sigma_{i} \sigma_{k} ; \sigma_{i} \sigma_{k}\right)-\left(\mu \rightarrow \mu^{\prime}\right)\right]_{C} \\
= & {\left[\left(\mu \sigma_{B}\left(\sigma_{i} \sigma_{k}\right)^{2}-\mu^{\prime} \sigma_{B}\left(\sigma_{i} \sigma_{k}\right)^{2}\right)-\left(\mu \sigma_{B} \sigma_{i} \sigma_{k}-\mu^{\prime} \sigma_{B} \sigma_{i} \sigma_{k}\right) 2 \mu \sigma_{i} \sigma_{k}\right.} \\
& -\left(\mu \sigma_{B}-\mu^{\prime} \sigma_{B}\right)\left(\mu\left(\sigma_{i} \sigma_{k} ; \sigma_{i} \sigma_{k}\right)-\left(\mu \sigma_{i} \sigma_{k}\right)^{2}\right) \\
& -\left(\mu\left(\sigma_{i} \sigma_{k}\right)^{2}-\mu^{\prime}\left(\sigma_{i} \sigma_{k}\right)^{2}\right) \mu \sigma_{B}-\left(\mu \sigma_{i} \sigma_{k}-\mu^{\prime} \sigma_{i} \sigma_{k}\right) \\
& \left.\times\left(\mu^{\prime} \sigma_{B} \sigma_{i} \sigma_{k}-\mu^{\prime} \sigma_{B} \mu \sigma_{i} \sigma_{k}+\mu^{\prime}\left(\sigma_{B} ; \sigma_{i} \sigma_{k}\right)+\mu^{\prime} \sigma_{B}\left(\mu \sigma_{i} \sigma_{k}+\mu^{\prime} \sigma_{i} \sigma_{k}\right)\right)\right]_{C} .
\end{aligned}
$$

Using that $\pm 2 a b \leqq a^{2}+b^{2}$ and $\left|\sigma_{A}\right| \leqq 1$, hence $\left|\left[\mu^{\left({ }^{\prime}\right)} \sigma_{A}\right]_{C}\right| \leqq 1$, for arbitrary $A$, we can, therefore, estimate the second term on the r.h.s. of (A.1) by

$$
\begin{aligned}
& \mid 2\left[\left(\mu \sigma_{B}-\mu^{\prime} \sigma_{B}\right) \partial_{i k}\left(\mu\left(\sigma_{B} ; \sigma_{i} \sigma_{k}\right)-\mu^{\prime}\left(\sigma_{B} ; \sigma_{i} \sigma_{k}\right)\right)\right]_{C} \\
& \leqq {\left[\left(\mu \sigma_{B}\left(\sigma_{i} \sigma_{k}\right)^{2}-\mu^{\prime} \sigma_{B}\left(\sigma_{i} \sigma_{k}\right)^{2}\right)^{2}+4\left(\mu \sigma_{B} \sigma_{i} \sigma_{k}-\mu^{\prime} \sigma_{B} \sigma_{i} \sigma_{k}\right)^{2}\right.} \\
& \quad\left.+19\left(\mu \sigma_{B}-\mu^{\prime} \sigma_{B}\right)^{2}+\left(\mu\left(\sigma_{i} \sigma_{k}\right)^{2}-\mu^{\prime}\left(\sigma_{i} \sigma_{k}\right)^{2}\right)^{2}+18\left(\mu \sigma_{i} \sigma_{k}-\mu^{\prime} \sigma_{i} \sigma_{k}\right)^{2}\right]_{C} .
\end{aligned}
$$

Combining (A.2) and (A.4), we find

$$
\begin{aligned}
\left|\partial_{i k}^{2}\left[\left(\mu \sigma_{B}-\mu^{\prime} \sigma_{B}\right)^{2}\right]_{C}\right| & \\
\leqq & K\left[\mu \sigma_{B}\left(\sigma_{i} \sigma_{k}\right)^{2}-\mu^{\prime} \sigma_{B}\left(\sigma_{i} \sigma_{k}\right)^{2}\right)^{2}+\left(\mu \sigma_{B} \sigma_{i} \sigma_{k}-\mu^{\prime} \sigma_{B} \sigma_{i} \sigma_{k}\right)^{2} \\
& \left.\quad+\left(\mu \sigma_{B}-\mu^{\prime} \sigma_{B}\right)^{2}+\left(\mu\left(\sigma_{i} \sigma_{k}\right)^{2}-\mu^{\prime}\left(\sigma_{i} \sigma_{k}\right)^{2}\right)^{2}+\left(\mu \sigma_{i} \sigma_{k}-\mu^{\prime} \sigma_{i} \sigma_{k}\right)^{2}\right]_{C}
\end{aligned}
$$

for some finite constant $K$.

For the Ising model, we may use that $\left(\sigma_{i} \sigma_{k}\right)^{2}=1$, and hence (A.5) yields (2.13). [Further simplifications arise for even, e.g. 0-, b.c., $|B|$ odd, and $d \varrho(\sigma)$ even in $\sigma$.] The important features of (A.5) are: 
(1) $K$ is a purely combinatorial constant independent of the specific model.

(2) All terms on the r.h.s. of (A.5) have the structure $\left[\left(\mu F-\mu^{\prime} F\right)^{2}\right]_{C}$, for some function $F$ of the spins $\sigma$ (and, for Ising- or $N$-vector models, $|F| \leqq 1$ ).

\section{Appendix A.2. Proof of Inequalities (2.54) and (3.51)}

By long, but elementary calculations, one finds, setting $\mu_{\Lambda \underline{g}}(\cdot) \equiv \mu(\cdot)$, that

$$
\begin{aligned}
\partial_{i k}\left[\mu\left(\sigma_{A} ; \sigma_{B}\right)^{2}\right]_{C}= & 2\left[\mu\left(\sigma_{A} \sigma_{i} \sigma_{k} ; \sigma_{B}\right)-\mu\left(\sigma_{A} ; \sigma_{B}\right) \mu \sigma_{i} \sigma_{k}-\mu\left(\sigma_{i} \sigma_{k} ; \sigma_{B}\right) \mu \sigma_{A}\right. \\
& +\mu\left(\sigma_{A} ; \sigma_{B}\right)\left\{\mu\left(\sigma_{A}\left(\sigma_{i} \sigma_{k}\right)^{2} ; \sigma_{B}\right)-\mu\left(\sigma_{i} \sigma_{k} ; \sigma_{B}\right) \mu \sigma_{A} \sigma_{i} \sigma_{k}\right. \\
& -[\ldots] \mu \sigma_{i} \sigma_{k}-\mu\left(\sigma_{A} ; \sigma_{B}\right) \mu\left(\sigma_{i} \sigma_{k} ; \sigma_{i} \sigma_{k}\right)-\mu\left(\left(\sigma_{i} \sigma_{k}\right)^{2} ; \sigma_{B}\right) \mu \sigma_{A} \\
& \left.\left.+\mu\left(\sigma_{i} \sigma_{k} ; \sigma_{B}\right)\left(\mu \sigma_{A}-\mu\left(\sigma_{A} ; \sigma_{i} \sigma_{k}\right)\right)\right\}\right]_{C} .
\end{aligned}
$$

Using inequalities like $\pm 2 a b \leqq a^{2}+b^{2},(a+b+c)^{2} \leqq 3\left(a^{2}+b^{2}+c^{2}\right),\left|\sigma_{D}\right| \leqq 1$, for arbitrary $D$, we get

$$
\begin{aligned}
\mid \partial_{i k}^{2}[ & \left.\mu\left(\sigma_{A} ; \sigma_{B}\right)^{2}\right]_{C} \mid \\
\leqq & K^{\prime}\left[\mu\left(\sigma_{A} ; \sigma_{B}\right)^{2}+\mu\left(\sigma_{A} \sigma_{i} \sigma_{k} ; \sigma_{B}\right)^{2}+\mu\left(\sigma_{A}\left(\sigma_{i} \sigma_{k}\right)^{2} ; \sigma_{B}\right)^{2}\right. \\
& \left.+\mu\left(\sigma_{i} \sigma_{k} ; \sigma_{B}\right)^{2}+\mu\left(\left(\sigma_{i} \sigma_{k}\right)^{2} ; \sigma_{B}\right)^{2}\right]_{C},
\end{aligned}
$$

for a purely combinatorial constant $K^{\prime}$. For the Ising model we may use $\left(\sigma_{i} \sigma_{k}\right)^{2}=1$, hence (A.7) yields (2.54).

We note that, on the r.h.s. of (A.7), all terms have the structure $\left[\mu(F ; G)^{2}\right]_{C}$, and in our models $|F| \leqq 1$ and $|G| \leqq 1$. This is nice, because if one wants to differentiate $\left[\mu(F ; G)^{2}\right]_{C}$ with respect to some variables $S_{l m},($ A.7) can be used again to bound the result.

Acknowledgements. We thank A. Bovier, J. Imbrie, and T. Kennedy for valuable comments and/or encouragement. B.Z. thanks the mathematics and physics departments of ETH-Zürich for hospitality during a period when this work was carried out.

\section{References}

1. Ruderman, M.A., Kittel, C.: Indirect exchange coupling of nuclear magnetic moments by conduction electrons. Phys. Rev. 96, 99 (1954)

2. Toulouse, G.: Commun. Phys. 2, 115 (1977)

3. Edwards, S.F., Anderson, P.W.: J. Phys. F 5, 965 (1975)

4. Little, W.A.: Math. Biosci. 19, 101 (1974)

Hopfield, J.J.: Proc. Natl. Acad. Sci. USA 79, 2554 (1982) and 81, 3088 (1984)

Hertz, J.: What are spin glasses and what do they have to do with association and memory, anyway? Lecture Notes, Nordita 1985

5. Kirkpatrick, S.: In: Lecture Notes in Physics, Vol. 149. Berlin, Heidelberg, New York: Springer 1981, p. 280

Kirkpatrick, S., Gelatt, C.D., Vecchi, M.P.: Science 220, 671 (1983)

Kirkpatrick, S., Toulouse, G.: Configuration space analysis of travelling salesman problems. J. Phys. (Paris) 46, 1277 (1985)

6. Sherrington, D., Kirkpatrick, S.: Solvable model of a spin-glass. Phys. Rev. Lett. 35, 1792 (1975)

7. Parisi, G.: Infinite number of order parameters for spin-glasses. Phys. Rev. Lett. 43, 1754 (1979) 
Parisi, G.: A sequence of approximated solutions to the $S-K$ model for spin glasses. J. Phys. A 13, L 115; The order parameter for spin glasses: A function on the interval 0-1. J. Phys. A 13, 1101; Magnetic properties of spin glasses in a new mean field theory. J. Phys. A 13, 1887 (1980) Parisi, G.: Order parameter for spin-glasses. Phys. Rev. Lett. 50, 1946 (1983)

Mézard, M., Parisi, G., Virasoro, M.: Europhys. Lett. 1, 77 (1986)

8. Bray, A.J., Moore, M.A.: Lower critical dimension of Ising spin glasses: A numerical study. J. Phys. C 17, L463 (1984)

Bray, A. J., Moore, M.A.: Critical behavior of the three-dimensional Ising spin glass. Phys. Rev. B 31, 631 (1985)

Mc Millan, W.L.: Domain-wall renormalization-group study of the three-dimensional random Ising model at finite temperature. Phys. Rev. B 31, 340 (1985); Domain-wall renormalization group study of the two-dimensional random Ising model. Phys. Rev. B 29, 4026 (1984)

Bhatt, R.N., Young, A.P.: Search for a transition in the three-dimensional $\pm J$ Ising spinglass. Phys. Rev. Lett. 54, 924 (1985)

Ogielski, A.T., Morgenstern, I.: Critical behavior of three-dimensional Ising spin-glass models. Phys. Rev. Lett. 54, 928 (1985)

Sourlas, N.: Three-dimensional Ising spin-glasses and mean field theory. Preprint E.N.S. 1984

9. Bovier, A., Fröhlich, J.: A heuristic theory of the spin glass phase. J. Stat. Phys. 44, 347 (1986) Bray, A.J., Moore, M.A.: Scaling theory of the ordered phase of spin glasses. To be publ. in Proc. of 1986 Heidelberg Colloquium on: Glassy dynamics and optimization. Lecture Notes in Physics; and refs. given there. Berlin, Heidelberg, New York: Springer

10. Fröhlich, J., Imbrie, J.: Improved perturbation expansion for disordered systems: Beating Griffiths singularities. Commun. Math. Phys. 96, 148 (1984)

Beretti, A.: Some properties of random Ising models. J. Stat. Phys. 38, 483 (1985)

11. Fröhlich, J., Zegarlinski, B.: The disordered phase of long-range Ising spin glasses. Europhys. Lett. 2, 53 (1986)

12. Khanin, K.M., Sinai, Ya.G.: Existence of free energy for models with long-range random Hamiltonians. J. Stat. Phys. 20, 573 (1979)

van Enter, A.C.D.: Spin glasses, effective decrease of long-range interactions. In: Proc. of Groningen conference on statistical mechanics. Hugenholtz, N.M., Winnink, M. (eds.). To appear; and refs. given there

13. Picco, P.: On the absence of breakdown of symmetry for the plane rotator model with longrange random interaction. J. Stat. Phys. 32, 627 (1983); Upper bound on the decay of correlations in the plane rotator model with long-range random interaction. J. Stat. Phys. 36, 489 (1984)

van Enter, A.C.D., Fröhlich, J.: Absence of symmetry breaking for $N$-vector spin glass models in two dimensions. Commun. Math. Phys. 98, 425 (1985)

14. Griffiths, R.B.: Nonanalytic behavior above the critical point in a random Ising ferromagnet. Phys. Rev. Lett. 23, 17 (1969)

15. Ruelle, D.: Probability estimates for continuous spin systems. Commun. Math. Phys. 50, 189 (1976)

16. Glimm, J., Jaffe, A., Spencer, T.: In: Constructive quantum field theory. Velo, G., Wightman, A.S. (eds.). Lecture Notes in Physics, Vol. 25. Berlin, Heidelberg, New York: Springer 1973

17. Ruelle, D.: Statistical mechanics (rigorous results). London, Amsterdam, Tokyo: W. A. Benjamin 1969

18. Brydges, D., Federbush, P.: In: Rigorous atomic and molecular physics. Velo, G., Wightman, A.S. (eds.). New York, London: Plenum Press 1981

19. Battle, G.A., III, Federbush, P.: A phase cell cluster expansion for a hierarchical $\Phi_{3}^{4}$ model. Commun. Math. Phys. 88, 263 (1983); Ondelettes and phase cell cluster expansions, a vindication. Commun. Math. Phys. (to appear)

Communicated by T. Spencer

Received September 15, 1986 
